This item was submitted to Loughborough's Research Repository by the author.

Items in Figshare are protected by copyright, with all rights reserved, unless otherwise indicated.

\title{
Spectra of Sol-manifolds: arithmetic and quantum monodromy
}

PLEASE CITE THE PUBLISHED VERSION

LICENCE

CC BY-NC-ND 4.0

\section{REPOSITORY RECORD}

Bolsinov, Alexey V., Holger R. Dullin, and A.P. Veselov. 2019. "Spectra of Sol-manifolds: Arithmetic and Quantum Monodromy”. figshare. https://hdl.handle.net/2134/397. 


\title{
SPECTRA OF Sol-MANIFOLDS: ARITHMETIC AND QUANTUM MONODROMY
}

\author{
A.V. BOLSINOV, H.R. DULLIN, AND A.P. VESELOV
}

\begin{abstract}
The spectral problem of three-dimensional manifolds $M_{A}^{3}$ admitting Sol-geometry in Thurston's sense is investigated. Topologically $M_{A}^{3}$ are torus bundles over a circle with a unimodular hyperbolic gluing map $A$. The eigenfunctions of the corresponding Laplace-Beltrami operators are described in terms of the modified Mathieu functions. It is shown that the multiplicities of the eigenvalues are the same for generic values of the parameters in the metric and are directly related to the number of representations of an integer by a given indefinite binary quadratic form. As a result the spectral statistics is shown to disagree with the Berry-Tabor conjecture. The topological nature of the monodromy for both classical and quantum systems on Sol-manifolds is demonstrated.
\end{abstract}

\section{INTRODUCTION}

It has been known since the nineteenth century that in dimension two there is a close relationship between geometry and topology. Namely each compact orientable surface admits a metric of constant curvature: positive if it is a topological sphere, zero if it is a torus and negative if it has genus more than 1 .

In dimension three the situation is much more sophisticated. The major development here was due to Thurston [26] who put forward the famous Geometrisation Conjecture: any compact orientable 3-manifold can be cut by disjoint embedded 2 -spheres and tori into pieces, which after gluing 3-balls to all boundary spheres, admit one of 8 special geometric structures. These special 3-dimensional geometries are the standard Euclidean $E^{3}$, spherical $S^{3}$ and hyperbolic $H^{3}$ geometries, the product geometries $S^{2} \times \mathbb{R}$ and $H^{2} \times \mathbb{R}$ and three geometries related to the Lie groups $S L_{2}(\mathbb{R}), N i l$ and $S o l$.

The last group $S o l$ is the 3-dimensional solvable Lie group, which is isomorphic to the group of isometries of Minkowski 2-space. The corresponding metric has the least symmetry of all the 8 geometries as the identity component of the stabiliser of a point is trivial.

The structure of 3-manifolds admitting any of the seven geometries excluding the most complicated hyperbolic case $H^{3}$ is pretty well understood. In particular a 3 -manifold $M$ possesses $S o l$-geometric structure if and only if $M$ is finitely covered by a torus bundle over $S^{1}$ with hyperbolic gluing map. For all other 6 geometries $M$ must be a Seifert fibre space (see e.g. [24]), so the Sol-manifolds are special from this point of view.

Their special role in the theory of dynamical systems became clear after a recent paper [4] by Taimanov and one of the authors, who showed the surprising fact that although the geodesic flow on Sol-manifolds is integrable in the sense of Liouville (but not in the analytic category) it has non-zero topological entropy! 
In the present paper we investigate the quantum version of the geodesic flow on Sol-manifolds, which is the spectral problem for the corresponding LaplaceBeltrami operator $\Delta$. We describe the spectra explicitly in terms of the spectrum of the modified Mathieu equation. These spectra are degenerate and have very interesting arithmetic. The multiplicities are directly related to the numbers of representations of a given integer by an indefinite binary quadratic form determined by the corresponding hyperbolic gluing map. This allows us to conclude that the spectral statistics for Sol-manifolds is not Poisson contrary to the well-known BerryTabor conjecture.

Note that the Sol-structure on Sol-manifolds is not unique in the same way as the flat structure on a torus is. The spectra of tori are very sensitive to a change of the flat metric: if we change the periods slightly the degeneracy will essentially disappear. The fact that this does not happen with Sol-manifolds shows the rigidity of the spectra and can be considered as a reflection of the hyperbolicity hidden inside the topology of Sol-manifolds.

We should mention that a deep relation of Sol-manifolds with arithmetic was known before (see e.g. [1, 5, 12]). In particular, Hirzebruch [12] and Atiyah, Donnelly and Singer [1] discovered a remarkable relation between topological "signature defects" of Sol-manifolds and arithmetical $L$-functions.

From the dynamical point of view the arithmetic and topology reveal themselves through Hamiltonian monodromy [8]. Its quantum analogue - quantum monodromy - is a relatively new phenomenon $[7,11,27]$, which still needs better understanding. An interesting feature of our case is that the corresponding grid of the quantum states can be described explicitly and nicely visualised ("Sol-flower", see fig. 6, 7 below). This is probably the first example of quantum monodromy of that kind.

The structure of the paper is following. First we introduce the class of Solmanifolds and describe the classical geodesic dynamics and the corresponding Hamiltonian monodromy. Then we review the facts from classical number theory about the relations between binary quadratic forms and the modular group $S L(2, \mathbb{Z})$. In section 5 we consider the spectral problem for the corresponding Laplace-Beltrami operator and find the eigenfunctions in terms of the modified Mathieu functions. The arithmetic of the multiplicities of the eigenvalues is discussed in detail in section 6 . The semiclassical analysis of the problem is done in section 7 in relation with Weyl's law. In section 8 we discuss the spectral statistics in the context of the Berry-Tabor conjecture [2]. The quantum monodromy for Sol-manifolds is discussed in the final section.

\section{Sol-MANIFOLDS}

In this paper we restrict ourselves to the main class of Sol-manifolds, which are $T^{2}$ torus bundles over a circle $S^{1}$ with hyperbolic gluing maps with positive eigenvalues. More precisely, consider the action of $\mathbb{Z}$ on $\tilde{M}^{3}=T^{2} \times \mathbb{R}$ generated by the following transformation $T_{A}$. Let $(x, y)$ be standard periodic coordinates on $T^{2}$ defined modulo 1 , and $z \in(-\infty,+\infty)$ be a coordinate on $\mathbb{R}$. Then in these coordinates the transformation $T_{A}$ is given by

$$
T_{A}:\left(\begin{array}{c}
x \\
y \\
z
\end{array}\right) \longrightarrow\left(\begin{array}{c}
a_{11} x+a_{12} y \\
a_{21} x+a_{22} y \\
z+1
\end{array}\right)
$$


where $A=\left(\begin{array}{ll}a_{11} & a_{12} \\ a_{21} & a_{22}\end{array}\right) \in S L(2, \mathbb{Z})$ is an integer hyperbolic matrix, which defines a hyperbolic automorphism of the 2-torus. The corresponding $S o l$-manifold $M_{A}^{3}$ is defined as the quotient $\tilde{M}^{3} / \mathbb{Z}$ by this action.

Let $\lambda$ and $\lambda^{-1}$ be the eigenvalues of $A$ and we assume that $\lambda>1$. The Solmanifolds with negative $\lambda$ are covered by those with positive eigenvalues.

Together with $(x, y, z)$ we shall use another coordinate system $(u, v, z)$ on $M_{A}^{3}$, where $(u, v)$ are linear coordinates on the fibres related to a positively oriented eigenbasis of $A$. The transformation $T_{A}$ in these coordinates is given by

$$
\left(\begin{array}{c}
u \\
v \\
z
\end{array}\right) \longrightarrow\left(\begin{array}{c}
\lambda u \\
\lambda^{-1} v \\
z+1
\end{array}\right)
$$

One should note that unlike $(x, y)$, the new coordinates $(u, v)$ are not periodic on the tori $T^{2}$ anymore: two pairs $(u, v),\left(u^{\prime}, v^{\prime}\right)$ define the same point on $T^{2}$ if and only if $\left(u-u^{\prime}, v-v^{\prime}\right)=k\left(c_{1}^{1}, c_{1}^{2}\right)+m\left(c_{2}^{1}, c_{2}^{2}\right)$, where $k, m \in \mathbb{Z}$ and $e_{1}=\left(c_{1}^{1}, c_{1}^{2}\right)$, $e_{2}=\left(c_{2}^{1}, c_{2}^{2}\right)$ is the basis of the lattice $\Gamma$ associated to $T^{2}$ :

$$
A=\left(\begin{array}{ll}
a_{11} & a_{12} \\
a_{21} & a_{22}
\end{array}\right)=\left(\begin{array}{cc}
c_{1}^{1} & c_{2}^{1} \\
c_{1}^{2} & c_{2}^{2}
\end{array}\right)^{-1}\left(\begin{array}{cc}
\lambda & 0 \\
0 & \lambda^{-1}
\end{array}\right)\left(\begin{array}{ll}
c_{1}^{1} & c_{2}^{1} \\
c_{1}^{2} & c_{2}^{2}
\end{array}\right)
$$

The Riemannian metrics on Sol-manifolds come from right-invariant metrics on the universal covering of $M_{A}^{3}$, which has the natural structure of a solvable Lie group Sol. Topologically this group is $\mathbb{R}^{3}$ with a multiplication of the form

$$
(u, v, w) *\left(u^{\prime}, v^{\prime}, w^{\prime}\right)=\left(u+e^{w} u^{\prime}, v+e^{-w} v^{\prime}, w+w^{\prime}\right) .
$$

One can realise it as the group of $3 \times 3$ matrices of the form

$$
\left(\begin{array}{ccc}
e^{w} & 0 & u \\
0 & e^{-w} & v \\
0 & 0 & 1
\end{array}\right) .
$$

The Sol-manifolds $M_{A}^{3}$ we consider are the quotients of the group Sol by the discrete subgroups $G_{A}$ corresponding to $w=m \ln \lambda, m \in \mathbb{Z}$ and $(u, v)=k e_{1}+l e_{2}$ belonging to the integer lattice $\Gamma$ described above, $z=w / \ln \lambda$.

The right-invariant metrics on the group Sol correspond to the following class of metrics on the $S o l$-manifold $M_{A}$ :

$$
\mathrm{d} s^{2}=\alpha(z) \mathrm{d} x^{2}+2 \beta(z) \mathrm{d} x \mathrm{~d} y+\gamma(z) \mathrm{d} y^{2}+\mathrm{d} z^{2}
$$

where

$$
\left(\begin{array}{cc}
\alpha(z) & \beta(z) \\
\beta(z) & \gamma(z)
\end{array}\right)=\exp (-z B)^{\top}\left(\begin{array}{cc}
\alpha & \beta \\
\beta & \gamma
\end{array}\right) \exp (-z B) .
$$

Here $\alpha, \beta, \gamma$ are real parameters with the only condition that the form $\mathrm{d} s^{2}=\alpha \mathrm{d} x^{2}+$ $2 \beta \mathrm{d} x \mathrm{~d} y+\gamma \mathrm{d} y^{2}$ is positive definite and $B$ is defined by the relation $\exp B=A$ :

$$
B=\left(\begin{array}{ll}
c_{1}^{1} & c_{2}^{1} \\
c_{1}^{2} & c_{2}^{2}
\end{array}\right)^{-1}\left(\begin{array}{cc}
\ln \lambda & 0 \\
0 & -\ln \lambda
\end{array}\right)\left(\begin{array}{ll}
c_{1}^{1} & c_{2}^{1} \\
c_{1}^{2} & c_{2}^{2}
\end{array}\right)
$$

One can consider a more general metric allowing a constant coefficient at $\mathrm{d} z^{2}$ but this will lead only to a general scaling. 


\section{Geodesic flows on Sol-manifolds: integrals and Hamiltonian MONODROMY}

Thus, the Hamiltonian of the geodesic flow on $M_{A}^{3}$ in $(u, v, z)$-coordinates can be written as

$$
H=\frac{1}{2}\left(E e^{2 z \ln \lambda} p_{u}^{2}+2 F p_{u} p_{v}+G e^{-2 z \ln \lambda} p_{v}^{2}\right)+\frac{1}{2} p_{z}^{2},
$$

where $E, F, G$ are real parameters: $E>0, G>0, E G-F^{2}>0$. It is invariant under the following transformation

$$
T_{A}^{*}:\left(\begin{array}{c}
u \\
v \\
z \\
p_{u} \\
p_{v} \\
p_{z}
\end{array}\right) \longrightarrow\left(\begin{array}{c}
\lambda u \\
\lambda^{-1} v \\
z+1 \\
\lambda^{-1} p_{u} \\
\lambda p_{v} \\
p_{z}
\end{array}\right)
$$

and, of course, under the translations by the elements of the lattice $\Gamma$. The same property must be satisfied for any smooth function on $T^{*} M_{A}^{3}$, in particular, for the first integrals of the geodesic flow.

Since $H$ depends neither on $u$, nor on $v$, the corresponding momenta $p_{u}$ and $p_{v}$ are local first integrals of the geodesic flow. However, being not invariant under (4), they are not well defined on the cotangent bundle $T^{*} M_{A}^{3}$. That is why, to get global first integrals, we need to replace $p_{u}, p_{v}$ by two smooth functions $f_{1}\left(p_{u}, p_{v}\right), f_{2}\left(p_{u}, p_{v}\right)$ invariant under the transformation $\left(p_{u}, p_{v}\right) \rightarrow\left(\lambda^{-1} p_{u}, \lambda p_{v}\right)$ (or, speaking in more general terms, by the invariants of the $\mathbb{Z}$-action on the cotangent plane generated by the hyperbolic linear transformation $A^{\top^{-1}}$ ).

One invariant function is evident: $Q=p_{u} p_{v}$. To find another one we introduce the following expression which will be useful also in the future

$$
\alpha=\frac{\ln \left(\sqrt{\frac{E}{G}}\left|\frac{p_{u}}{p_{v}}\right|\right)}{2 \ln \lambda} .
$$

Under the transformation (4) $\alpha$ changes in a very simple way:

$$
\alpha\left(p_{u}, p_{v}\right) \rightarrow \alpha\left(p_{u}, p_{v}\right)-1
$$

Thus, as a second integral we can take any function of $\alpha$ with period 1 , for instance, $\cos (2 \pi \alpha)$ or $\sin (2 \pi \alpha)$. However these functions are not smooth at $p_{u}=$ $p_{v}=0$. To avoid this difficulty and to get the first integrals in a more symmetric form we put:

$$
\begin{aligned}
& f_{1}=R(Q) \cos 2 \pi \alpha, \\
& f_{2}=R(Q) \sin 2 \pi \alpha,
\end{aligned}
$$

where

$$
R(Q)=\sqrt{|Q|} \exp \left(-\frac{1}{Q^{2}}\right)
$$

Remark. The fact that the second integral is not analytic is not accidental: the theorem proved by Taimanov [25] implies that Sol-manifolds do not admit integrable geodesic flows with analytic integrals (see [4] for more details).

We are going to show now that one can see the topological structure of the Solmanifolds by looking at the Hamiltonian monodromy of the geodesic flow. For that 
we will have to investigate the bifurcation diagram (i.e. the set of critical values) of the momentum mapping restricted to the isoenergy surface $E_{A}^{5}=\{H=1\}$ :

$$
\mathcal{F}_{A}=\left(f_{1}, f_{2}\right): E_{A}^{5} \rightarrow \mathbb{R}^{2} .
$$

Proposition 1. The bifurcation diagram of the momentum mapping $\mathcal{F}_{A}$ consists of two circles

$$
f_{1}^{2}+f_{2}^{2}=R^{2}\left(Q_{ \pm}^{*}\right)
$$

where $Q_{ \pm}^{*}=(F \pm \sqrt{E G})^{-1}$, and the point $(0,0)$, the centre of these circles. The set of critical points consists of five parts: a) four one-parameter families $L_{i}(i=$ $1, \ldots, 4)$ of (degenerate) 2-dimensional tori lying in the cotangent bundle and given by ( $\alpha$ is a parameter):

$$
z=-\alpha, u \text { and } v \text { are arbitrary, }
$$

$$
p_{z}=0, \quad p_{u}= \pm \sqrt{\frac{e^{2 \alpha \ln \lambda}}{E\left(1+\frac{F}{\sqrt{E G}}\right)}}, \quad p_{v}= \pm \sqrt{\frac{e^{-2 \alpha \ln \lambda}}{G\left(1+\frac{F}{\sqrt{E G}}\right)}} ;
$$

and

$$
z=-\alpha, u \text { and } v \text { are arbitrary, }
$$

$$
p_{z}=0, \quad p_{u}= \pm \sqrt{\frac{e^{2 \alpha \ln \lambda}}{E\left(1-\frac{F}{\sqrt{E G}}\right)}} \quad p_{v}=\mp \sqrt{\frac{e^{-2 \alpha \ln \lambda}}{G\left(1-\frac{F}{\sqrt{E G}}\right)}}
$$

b) the critical set $N$ given by the equation $Q=p_{u} p_{v}=0$.

Proof. We are interested in the singularities of $\mathcal{F}_{A}$ or, which is the same, those of the Liouville foliation. These singularities can be of two types. To explain their nature we first consider the geodesic flow on the covering manifold $\tilde{M}^{3}$. On this (non-compact) manifold the integrals of the flow are simply $p_{u}$ and $p_{v}$. Consider the Liouville foliation for this covering system. Its singular leaves correspond to the critical points of the momentum mapping

$$
\tilde{\mathcal{F}}=\left(p_{u}, p_{v}\right): \tilde{E}^{5} \rightarrow \mathbb{R}^{2},
$$

where $\tilde{E}^{5}=\{H=1\} \subset T^{*} \tilde{M}$. Obviously, these leaves remain singular after the natural projection $\tilde{E}^{5} \rightarrow E_{A}^{5}$. These are singularities of the first type.

On the other hand some new singularities appear since instead of $p_{u}$ and $p_{v}$ we have to consider more complicated functions $f_{1}$ and $f_{2}$. In other words, these are singularities of the map $\left(p_{u}, p_{v}\right) \rightarrow\left(f_{1}, f_{2}\right)$.

Let us treat both cases in turns. It is easily seen that $p_{u}$ and $p_{v}$ are functionally dependent, as functions on $\tilde{E}^{5}=\{H=1\}$ if and only if two conditions are simultaneously satisfied: 1) $\frac{\partial H}{\partial p_{z}}=2 p_{z}=0$ and 2) $\frac{\partial H}{\partial z}=0$. Taking into account the condition $H=1$, we obtain a system of equations

$$
\begin{aligned}
& E e^{2 \log \lambda z} p_{u}^{2}-G e^{-2 \log \lambda z} p_{v}^{2}=0, \\
& E e^{2 \log \lambda z} p_{u}^{2}+2 F p_{u} p_{v}+G e^{-2 \log \lambda z} p_{v}^{2}=2
\end{aligned}
$$

The first equation gives

$$
z=-\frac{\ln \left(\sqrt{\frac{E}{G}}\left|\frac{p_{u}}{p_{v}}\right|\right)}{2 \ln \lambda}=-\alpha .
$$




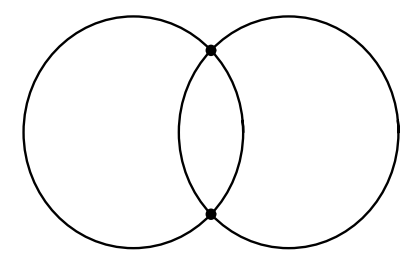

Figure 1. The topological structure of the singular leaf is $M_{A}^{3} \times K$ where $K$ is given in the figure.

Now solving this system with respect $p_{u}$ and $p_{v}$ (after substituting $z=-\alpha$ ), we find four distinct solutions:

$$
\begin{array}{ll}
\text { 1) } p_{u}=\frac{e^{\alpha \ln \lambda}}{\sqrt{E\left(1+\frac{F}{\sqrt{E G}}\right)}}, & p_{v}=\frac{e^{-\alpha \ln \lambda}}{\sqrt{G\left(1+\frac{F}{\sqrt{E G}}\right)}} ; \\
\text { 2) } p_{u}=\frac{-e^{\alpha \ln \lambda}}{\sqrt{E\left(1+\frac{F}{\sqrt{E G}}\right)}}, & p_{v}=\frac{-e^{-\alpha \ln \lambda}}{\sqrt{G\left(1+\frac{F}{\sqrt{E G}}\right)}} ; \\
\text { 3) } p_{u}=\frac{e^{\alpha \ln \lambda}}{\sqrt{E\left(1-\frac{F}{\sqrt{E G}}\right)}}, & p_{v}=\frac{-e^{-\alpha \ln \lambda}}{\sqrt{G\left(1-\frac{F}{\sqrt{E G}}\right)}} ; \\
\text { 4) } p_{u}=\frac{-e^{\alpha \ln \lambda}}{\sqrt{E\left(1-\frac{F}{\sqrt{E G}}\right)}}, & p_{v}=\frac{e^{-\alpha \ln \lambda}}{\sqrt{G\left(1-\frac{F}{\sqrt{E G}}\right)}}
\end{array}
$$

Thus, for each value of $\alpha$ we obtain four 2-dimensional invariant tori in $T^{*} M_{A}^{3}$. All of them are diffeomorphically projected onto the same $T^{2}$-fibre $T_{-\alpha}^{2}=\{z=$ const $=-\alpha\} \subset M$. Varying $\alpha$, we obtain 4 families of degenerate Liouville 2-tori $L_{i}, i=1, \ldots, 4$.

It is easy to verify that for each family $L_{i}$ the value of $Q=p_{u} p_{v}$ is constant and equal to $Q_{+}^{*}=(F+\sqrt{E G})^{-1}$ for $L_{1}$ and $L_{2}$, and equal to $Q_{-}^{*}=(F-\sqrt{E G})^{-1}$ for $L_{3}$ and $L_{4}$. Hence the image of $L_{1}$ and $L_{2}$ is the circle $f_{1}^{2}+f_{2}^{2}=R^{2}\left(Q_{+}^{*}\right)$, and analogously the image of $L_{3}$ and $L_{4}$ is the other circle $f_{1}^{2}+f_{2}^{2}=R^{2}\left(Q_{-}^{*}\right)$, as required.

The singularities of the second type come from those of the mapping $\left(p_{u}, p_{v}\right) \rightarrow$ $\left(f_{1}, f_{2}\right)$. It can be easily seen that the critical points of this mapping are defined by the equation $Q=p_{u} p_{v}=0$. This implies immediately $f_{1}=f_{2}=0$ which gives a single point on the bifurcation diagram, namely the centre of the circles.

Notice that topologically the subset $N=\left\{Q=p_{u} p_{v}=0\right\} \subset T^{*} M_{A}^{3}$ is homeomorphic to the direct product $M_{A}^{3} \times K$, where $K$ is a graph that consists of two vertices and four segments connecting them (see fig. 1). This follows immediately from the parallelizability of $M_{A}^{3}$ and the simple observation that in each cotangent space the conditions $p_{u} p_{v}=0, H=1$ define a graph homeomorphic to $K$.

Now we are able to describe the global structure of the foliation of the isoenergy surface $E_{A}^{5}$ into Liouville tori.

If we remove the singular set from the isoenergy surface we obtain four families of 3-dimensional Liouville tori distinguished from each other by signs of $p_{u}$ and $p_{v}$ :
a) $p_{u}>0, p_{v}>0$
b) $p_{u}<0, p_{v}<0$
c) $p_{u}>0, p_{v}<0$
d) $p_{u}<0, p_{v}>0$ 
The families a) and b) are isomorphic (more precisely, they transform into each other by the globally defined time reversal automorphism of the geodesic flow $\left.\left(u, v, z, p_{u}, p_{v}, p_{z}\right) \rightarrow\left(u, v, z,-p_{u},-p_{v},-p_{z}\right)\right)$.

The same is true for the families c) and d).

Each Liouville 3 -torus is uniquely determined by the values of two integrals $Q$ and $\alpha \bmod 1$, where the values of $Q$ form the interval $\left(0, Q_{+}^{*}\right)$ in the first two cases and $\left(Q_{-}^{*}, 0\right)$ for the other two cases. In particular, in each of the cases, the base of the $T^{3}$-foliation is homeomorphic to a punctured disc. As $Q \rightarrow 0$, the Liouville torus approaches the singular set $N$. As $Q \rightarrow Q_{ \pm}^{*}$, the torus shrinks into one of the degenerate 2-tori described above.

Thus, the base of the global Liouville foliation on $E_{A}^{5}=\{H=1\}$ can be considered as four discs glued together at their centres. All interior points of these discs except the centre correspond one-to-one to regular 3-dimensional Liouville tori, the boundary circles of the discs correspond to the families $L_{i}$ of degenerate 2-tori, and finally, the common center of the discs corresponds to the singular set $N$.

The image of each family under the momentum map is a 2-disc with the center removed. This is exactly the situation when we can talk about Hamiltonian monodromy [8].

Theorem 1. For each family of Liouville 3-tori there exist a basis of cycles in the first homology group of the tori in which the Hamiltonian monodromy has the matrix

$$
\left(\begin{array}{cc}
A & 0 \\
0 & 1
\end{array}\right)
$$

Proof. This fact can be observed in many different ways. We shall follows the definition of Hamiltonian monodromy and will explicitely compute the deformation of Liouville tori and the final gluing map.

Consider an arbitrary Liouville 3 -torus $T^{3}=T_{Q_{0}, \alpha_{0}}^{3}$. In coordinates, this torus is given by three conditions:

$$
\begin{aligned}
& E e^{2 z \ln \lambda} p_{u}^{2}+2 F p_{u} p_{v}+G e^{-2 z \ln \lambda} p_{v}^{2}+p_{z}^{2}=2, \\
& Q\left(p_{u}, p_{v}\right)=p_{u} p_{v}=Q_{0} \\
& \alpha\left(p_{u}, p_{v}\right)=\frac{\ln \left(\sqrt{\frac{E}{G}}\left|\frac{p_{u}}{p_{v}}\right|\right)}{2 \ln \lambda}=\alpha_{0} \quad \bmod 1
\end{aligned}
$$

More precisely, these conditions define a disjoint union of two or four tori, which differ from each other by the signs of the momenta $p_{u}$ and $p_{v}$. We consider one of them $T_{Q_{0}, \alpha_{0}}^{3}$ by putting for definiteness $p_{u}>0, p_{v}>0$.

For our purposes first we need to explain why the above conditions define indeed a three-dimensional torus and to describe the basic cycles on this torus. Notice that the common level set (7) of the first integrals can be regarded from two slightly different points of view: as a subset in $T^{*} \tilde{M}^{3}$ and that in $T^{*} M_{A}^{3}$. However one can show that the natural projection $T^{*} \tilde{M}^{3} \rightarrow T^{*} M_{A}^{3}$ restricted to this level set is a diffeomorphism (no points are glued between them). Thus, in fact there is no real difference between these two points of view. In particular, instead of conditions $p_{u} p_{v}=Q_{0}, \alpha\left(p_{u}, p_{v}\right)=\alpha_{0} \bmod 1$ we may simply assume that the momenta $p_{u}, p_{v}$ themselves are constant. Then the conditions (7) can be rewritten as:

$$
p_{u}=\text { const }, \quad p_{v}=\text { const }, \quad u \text { and } v \text { are arbitrary, }
$$

and

$$
c_{1} \cosh \left(2 \ln \lambda\left(z+\alpha_{0}\right)\right)+p_{z}^{2}=c_{2},
$$


where $c_{1}=2 \sqrt{E G}\left|Q_{0}\right|, c_{2}=2-2 F Q_{0}$. We see that the variables separate and the fact that this systems defines a 3 -torus becomes evident. Indeed, the variables $u, v$ "run" over a two-dimensional torus and the last equation defines a simple closed curve on the plane $\mathbb{R}^{2}\left(z, p_{z}\right)$. In other words, we have a natural splitting of $T_{Q_{0}, \alpha_{0}}^{3}$ into the direct product $T^{2} \times S^{1}$. Thus, as basic cycles on $T_{Q_{0}, \alpha_{0}}^{3}$ we can take the cycles on $T^{2}(u, v)$ related to the original coordinate system $(x, y)$ (see above) and the third cycle defined by (8).

Now let us look at what happens to this torus if we change the parameters $Q_{0}$ and $\alpha_{0}$ in such a way that the point $\mathcal{F}_{A}\left(T_{Q_{0}, \alpha_{0}}^{3}\right)$ moves inside the image of the momentum mapping around the singular point $\mathcal{F}_{A}(N)=(0,0)$. It is easy to see that this deformation just means that we change the value of $\alpha$, while $Q$ can be chosen to remain constant:

$$
Q(t)=Q_{0}, \quad \alpha(t)=\alpha_{0}+t, \quad t \in[0,1] .
$$

Consider the family of mappings

$$
\phi_{t}\left(u, v, z, p_{u}, p_{v}, p_{z}\right)=\left(u, v, z-t, e^{t \ln \lambda} p_{u}, e^{-t \ln \lambda} p_{v}, p_{z}\right) .
$$

It is not hard to see that the image of $T_{Q_{0}, \alpha_{0}}^{3}$ under $\phi_{t}$ is exactly $T_{Q_{0}, \alpha_{0}+t}^{3}$ and $\phi_{t}: T_{Q_{0}, \alpha_{0}}^{3} \rightarrow T_{Q_{0}, \alpha_{0}+t}^{3}$ is a difeomorphism. In other words, $\phi_{t}$ defines the deformation of Liouville tori we need.

At the moment $t=1$ the torus comes back to the initial position, i.e., $T_{Q_{0}, \alpha_{0}}^{3}=$ $T_{Q_{0}, \alpha_{0}+1}^{3}$, and we obtain the monodromy map

$$
\phi_{1}: T_{Q_{0}, \alpha_{0}}^{3} \rightarrow T_{Q_{0}, \alpha_{0}}^{3}=T_{Q_{0}, \alpha_{0}+1}^{3}
$$

Now our goal is to describe the corresponding automorphism of the first homology group:

$$
\phi_{1_{*}}: H_{1}\left(T_{Q_{0}, \alpha_{0}}^{3}\right)=\mathbb{Z}^{3} \rightarrow H_{1}\left(T_{Q_{0}, \alpha_{0}}^{3}\right)=\mathbb{Z}^{3} .
$$

Using the identification (4) we see that the map $\phi_{t}$ can be rewritten as follows:

$$
\phi_{t}\left(\begin{array}{c}
u \\
v \\
z \\
p_{u} \\
p_{v} \\
p_{z}
\end{array}\right)=\left(\begin{array}{c}
\lambda u \\
\lambda^{-1} v \\
z \\
p_{u} \\
p_{v} \\
p_{z}
\end{array}\right)
$$

We see that the only transformation is related to the variables $u$ and $v$. Moreover, this transformation is exactly the original hyperbolic automorphism $A: T^{2} \rightarrow T^{2}$. Taking into account the natural splitting $T_{Q_{0}, \alpha_{0}}^{3}=T^{2}(u, v) \times S^{1}\left(z, p_{z}\right)$ we conclude immediately that the monodromy matrix in the chosen basis is

$$
\left(\begin{array}{cc}
A & 0 \\
0 & 1
\end{array}\right) \text {. }
$$

We conclude this section with the discussion of the geodesics on Sol-manifolds. They have different properties depending on the types of leaves of the Liouville foliation which they belong to.

First consider the geodesics lying on Liouville tori of dimension three. They are characterized by the property that all momenta $p_{u}, p_{v}$ and $p_{z}$ differ from zero. 
More precisely, the signs $p_{u}$ and $p_{v}$ always remain the same, whereas the sign of $p_{z}$ changes. This happens when $z$ reaches the value

$$
z_{ \pm}=\frac{ \pm \cosh ^{-1}\left(\frac{h-F p_{u} p_{v}}{\sqrt{E G}\left|p_{u} p_{v}\right|}\right)}{2 \ln \lambda}-\alpha\left(p_{u}, p_{v}\right) .
$$

Two levels $z=z_{+}$and $z=z_{-}$are exactly the caustics of the Liouville tori that contains a given geodesic. The situation is quite similar to that on a surface of revolution where the motion takes place between two levels of $z$.

It is easy to see that the distance between these levels $z_{+}-z_{-}$tends to infinity as $p_{u} p_{v}$ tends to zero. From this it follows that the corresponding geodesics rotate many times (along the base $S^{1}$ ), then turn back, after this go in the opposite direction, then turn back and so on. As $p_{u} p_{v}$ tends to zero the number of rotations in one direction until turning back (or, which is the same, between two caustics) increases up to infinity.

If $p_{u} p_{v}=0$, then we are on the singular level. The corresponding geodesics have the following behaviour. If both $p_{u}$ and $p_{v}$ vanish, then we obtain the family of geodesics

$$
u=\text { const }, \quad v=\text { const }, \quad z=t .
$$

Such geodesics obviously form an invariant submanifold $N_{+}$in $T^{*} M$ which is diffeomorphic to $M$. Exactly on this submanifold the geodesic flow is chaotic and has positive entropy. Indeed, the time-one map transform each fibre $T_{z}^{2}$ into itself by means of the hyperbolic automorphism $A$. As well known, the entropy of $A$ : $T^{2} \rightarrow T^{2}$ is $\ln \lambda>0$.

There is another invariant submanifold $N_{-}$with the same properties formed by vertical geodesics going in the opposite direction:

$$
u=\text { const }, \quad v=\text { const }, \quad z=-t .
$$

From the viewpoint of the ambient geodesic flow $N_{+}$and $N_{-}$are hyperbolic invariant subsets. The stable manifold coresponding to $N_{+}$is given by $p_{v}=0$, the unstable one is $p_{u}=0$. For $N_{-}$the stable and unstable manifolds interchange. The geodesics satisfying the condition $p_{v}=0$ as $t \rightarrow+\infty$ asymptocally approaches $N_{+}$, in particular, $p_{z} \rightarrow+1$. But there is $t=t_{0}$ when $p_{z}$ changes sign so that for $t \rightarrow-\infty$ the geodesic approaches to $N_{-}$. The geodesics satisfying $p_{u}=0$ behave in the opposite way.

In slightly other terms this structure can be described as follows: there are two hyperbolic submanifolds diffeomorphic to $M_{A}^{3}$, they are connected by 4 fourdimensional separatrices, see fig. 1

Finally we would like to mention an interesting phenomenon which one would not expect from an integrable geodesic flow on a compact manifold. Namely, one of the action integrals diverges as the integral $Q \rightarrow 0$ with the energy fixed (see the calculations and footnote in Section 7). Normal scenario would be when approaching the singular level some of the cycles of the Liouville tori shrink so the actions will stay finite. The fact that this not true for Sol-manifolds when one approaches the singular (chaotic) level demonstrates once again the peculiar nature of this system.

To discuss the quantum case we will need some facts from the classical number theory, which we present in the next section. 


\section{4. $S L(2, \mathbb{Z})$ AND BINARY QUADRATIC FORMS}

The content of this section is well-known (see e.g. [18, 19, 21]).

Let $A=\left(\begin{array}{ll}a_{11} & a_{12} \\ a_{21} & a_{22}\end{array}\right) \in S L(2, \mathbb{Z})$ be an integer hyperbolic matrix. Hyperbolicity as before means that its eigenvalues are real and distinct. We would like to consider $A$ as the automorphism of the lattice $\mathcal{L}=\mathbb{Z} \oplus \mathbb{Z} \in \mathbb{R}^{2}$ by choosing some basis $e_{1}, e_{2}$ in this lattice.

For any such $A$ we can define the following integer binary quadratic form $Q_{A}$ by the formula

$$
A \mathbf{v} \wedge \mathbf{v}=Q_{A}(\mathbf{v}) e_{1} \wedge e_{2},
$$

where $\mathbf{v}$ is a vector from $\mathbb{R}^{2}$. Explicitly if $\mathbf{v}=x e_{1}+y e_{2}$ then

$$
Q_{A}(x, y)=\operatorname{det}\left(\begin{array}{ll}
a_{11} x+a_{12} y & x \\
a_{21} x+a_{22} y & y
\end{array}\right)=-a_{21} x^{2}+\left(a_{11}-a_{22}\right) x y+a_{12} y^{2} .
$$

It is easy to see from the definition that this form is invariant under the action of $A$ :

$$
Q_{A}(A \mathbf{v})=Q_{A}(\mathbf{v}) .
$$

Notice that $Q_{A}$ has the discriminant

$D=\left(a_{11}-a_{22}\right)^{2}+4 a_{12} a_{21}=\left(a_{11}+a_{22}\right)^{2}-4\left(a_{11} a_{22}-a_{12} a_{21}\right)=\left(a_{11}+a_{22}\right)^{2}-4$,

which is exactly the discriminant of the characteristic equation of $A$ :

$$
\lambda^{2}-\left(a_{11}+a_{22}\right) \lambda+1=0 .
$$

In particular, since $A$ is hyperbolic the form $Q_{A}$ is indefinite. Note that the discriminant $D$ cannot be a total square.

In general the coefficients of the quadratic form $Q_{A}$ may have a common factor. Let

$$
\hat{Q}_{A}(x, y)=a x^{2}+b x y+c y^{2}
$$

be its primitive form after division of $Q_{A}$ by the largest common factor. It is defined correctly only up to a sign.

Thus to each integer unimodular hyperbolic matrix $A$ we relate an indefinite integer primitive quadratic form $\hat{Q}_{A}$.

Conversely, suppose we have such a form $Q(x, y)=a x^{2}+b x y+c y^{2}$. We would like to describe all $A$ from $S L(2, \mathbb{Z})$ which preserve this form. Such $A$ are called the automorphs of $Q$. Let

$$
d=b^{2}-4 a c
$$

be the discriminant of $Q$ which we assume not to be a total square and consider the corresponding Diophantine equation called Pell's equation:

$$
X^{2}-d Y^{2}=4 .
$$

Then the group of automorphs consists of matrices of the form

$$
A= \pm\left(\begin{array}{cc}
\frac{X-b Y}{2} & -c Y \\
a Y & \frac{X+b Y}{2}
\end{array}\right)
$$


where $(X, Y)$ are the solutions of Pell's equation. Modulo $\pm I$ this group is cyclic with generator

$$
A_{0}=\left(\begin{array}{cc}
\frac{X_{0}-b Y_{0}}{2} & -c Y_{0} \\
a Y_{0} & \frac{X_{0}+b Y_{0}}{2}
\end{array}\right),
$$

where $\left(X_{0}, Y_{0}\right)$ is the fundamental solution of this equation.

Recall that $\left(X_{0}, Y_{0}\right)$ is the fundamental solution of Pell's equation if $X_{0}>0, Y_{0}>$ 0 and $X_{0}+\sqrt{d} Y_{0}$ is minimal among all such solutions. The classical result about Pell's equation says that all other solutions can be found from the relation

$$
\frac{X+\sqrt{d} Y}{2}= \pm\left(\frac{X_{0}+\sqrt{d} Y_{0}}{2}\right)^{n},
$$

where $n=0,1, \ldots$ One can find the fundamental solution from the continued fraction of $\sqrt{d}$. This structure of the solutions of Pell's equations induces the cyclic group structure for the automorphs.

Notice that the form $Q_{A}$ corresponding to the matrix (13) has the form

$$
Q=Y_{0}\left(a x^{2}+b x y+c y^{2}\right) \text {. }
$$

Let us call a hyperbolic element $A$ from $S L(2, \mathbb{Z})$ primitive if it can not be represented as a power of any other element from $S L(2, \mathbb{Z})$.

Thus we have described a natural correspondence between the primitive binary indefinite forms $Q$ and primitive elements $A$ from $S L(2, \mathbb{Z})$. In particular, it helps us to answer the question if a given integer unimodular matrix $A$ is a primitive or if not which power of a primitive matrix it is.

\section{Spectrum and eigenfunctions of the Laplace-Beltrami operator}

Let us now discuss the quantum geodesic problem on the Sol-manifold $M_{A}^{3}$ :

$$
-\Delta \psi=\mathcal{E} \psi,
$$

where $\Delta$ is the Laplace-Beltrami operator on $M_{A}^{3}$ and $\psi=\psi(P, \mathcal{E}), P \in M_{A}^{3}$. In coordinates $(u, v, z)$ the Laplace-Beltrami operator has the following explicit form:

$$
\Delta=E e^{2 z \ln \lambda} \frac{\partial^{2}}{\partial u^{2}}+2 F \frac{\partial^{2}}{\partial u \partial v}+G e^{-2 z \ln \lambda} \frac{\partial^{2}}{\partial v^{2}}+\frac{\partial^{2}}{\partial z^{2}} .
$$

This is a self-adjoint operator in the Hilbert space $L_{2}\left(M_{A}^{3}\right)$ where the integration measure on $M_{A}^{3}$ is induced by the Riemannian metric (3). In both $(x, y, z)$ and $(u, v, z)$ coordinate systems the corresponding measure $d \mu$ is proportional to the standard Lebesgue measure on $\mathbb{R}^{3}$.

Because the coefficients of $\Delta$ depends only on $z$ it is quite natural to separate variables and look for the eigenfunctions of $\Delta$ of the form

$$
\Psi_{\gamma}(u, v, z)=e^{2 \pi i(\gamma, w)} f(z),
$$

where $\gamma$ is an element of the dual lattice $\Gamma^{*}$ corresponding to the $T^{2}$-fibres and $w=(u, v)$ (so the scalar product $(\gamma, w)$ is defined modulo $\mathbb{Z}$ ).

By substituting into the Schrödinger equation (14), (15) we get

$$
\Delta \Psi_{\gamma}=\left(\frac{\partial^{2} f}{\partial z^{2}}-8 \pi^{2} \sqrt{E G}|Q(\gamma)|\left(\cosh (2 \ln \lambda(z+\alpha(\gamma)))+\frac{F \operatorname{sgn} Q(\gamma)}{\sqrt{E G}}\right) f\right) e^{2 \pi i(\gamma, w)},
$$


where $Q(\gamma)=\left(\gamma, e_{u}\right)\left(\gamma, e_{v}\right)$ is a quadratic form on the lattice $\Gamma^{*}$, and

$$
\alpha(\gamma)=\frac{\ln \left(\sqrt{\frac{E}{G}}\left|\frac{\left(\gamma, e_{u}\right)}{\left(\gamma, e_{v}\right)}\right|\right)}{2 \ln \lambda} .
$$

Here $e_{u}$ and $e_{v}$ are the eigenvectors of $A$ related to the eigenvalues $\lambda$ and $\lambda^{-1}$ respectively and the basis $e_{u}, e_{v}$ is assumed to be positively oriented. Notice that $\alpha$ is the same as before in (5) if we replace $p_{u}$ by $\left(\gamma, e_{u}\right)$ and $p_{v}$ by $\left(\gamma, e_{v}\right)$.

To clarify the meaning of the coefficient in front of the cosh let us consider the basis $e_{u}^{*}, e_{v}^{*}$ in $\mathbb{R}^{2 *}$ dual to $e_{u}, e_{v}$. The vectors $e_{u}^{*}$ and $e_{v}^{*}$ are also the eigenvectors of $A^{*}$ with the eigenvalues $\lambda$ and $\lambda^{-1}$ respectively. By definition we have $\gamma=$ $\left(\gamma, e_{u}\right) e_{u}^{*}+\left(\gamma, e_{v}\right) e_{v}^{*}$. Since $Q(\gamma)$ is obviously invariant under the action of $A^{*}$ it is natural to compare it with the binary form $Q_{A^{*}}$ defined in the section 3 . We have

$$
\begin{aligned}
A^{*} \gamma \wedge \gamma & =\left(\lambda\left(\gamma, e_{u}\right) e_{u}^{*}+\lambda^{-1}\left(\gamma, e_{v}\right) e_{v}^{*}\right) \wedge\left(\left(\gamma, e_{u}\right) e_{u}^{*}+\left(\gamma, e_{v}\right) e_{v}^{*}\right) \\
& =\left(\gamma, e_{u}\right)\left(\gamma, e_{v}\right)\left(\lambda-\lambda^{-1}\right) e_{u}^{*} \wedge e_{v}^{*} .
\end{aligned}
$$

Let $l_{1}, l_{2}$ be a positively oriented basis in the dual lattice $\Gamma^{*}$, then by definition $A^{*} \gamma \wedge \gamma=Q_{A^{*}}(\gamma) l_{1} \wedge l_{2}$.

From these calculations and from the equalities $E=\left|e_{u}^{*}\right|^{2}, G=\left|e_{v}^{*}\right|^{2}$ it follows that

where

$$
\sqrt{E G}|Q(\gamma)|=c\left|Q_{A^{*}}(\gamma)\right|,
$$

$$
c=c(A ; E, F, G)=\frac{\mathcal{A}\left(\square^{*}\right)}{\sqrt{D} \sin \theta}=\frac{1}{\sqrt{D} \mathcal{A}\left(T^{2}\right) \sin \theta}
$$

$\mathcal{A}\left(\square^{*}\right)$ is the area of the dual basic parallelogram $\Pi\left(e_{1}^{*}, e_{2}^{*}\right)$ (which is the inverse of the area of the fibre $\left.T^{2}\right), D=\left(\lambda-\lambda^{-1}\right)^{2}$ is the discriminant of the characteristic equation of the matrix $A$ (or equivalently $A^{*}$ ), and $\theta$ is the angle between $e_{u}^{*}$ and $e_{v}^{*}$. Thus we have proved the following

Proposition 2. A function $\Psi=e^{2 \pi i(\gamma, w)} f(z)$ satisfies equation (14) if and only if $f(z)$ satisfies the modified Mathieu equation

$$
\left(-\frac{\mathrm{d}^{2}}{\mathrm{~d} z^{2}}+|\nu(\gamma)| \cosh 2 \mu(z+\alpha(\gamma)) f(z)=\Lambda f(z),\right.
$$

where $\mu=\ln \lambda, \nu(\gamma)=8 \pi^{2} c Q_{A^{*}}(\gamma)$ and $\alpha(\gamma)$ is given above. The eigenvalues $\mathcal{E}$ and $E$ are related by the shift

$$
\mathcal{E}=\Lambda+\nu(\gamma) \cos \theta
$$

Recall that the modified Mathieu equation is the cosh-version of the standard Mathieu equation

$$
\frac{\mathrm{d}^{2} y}{\mathrm{~d} x^{2}}+(a \cos 2 \mu x+b) y=0 .
$$

Its solutions are known as modified Mathieu functions (see e.g. [30, 31]). They appear also in the theory of Coulomb spheroidal functions [15], where one can find some related numerical results (see also [16]).

Let $\Lambda=\Lambda_{k}(\nu), k=1,2, \ldots$ be the spectrum of the corresponding modified Mathieu operator

$$
\mathcal{M}=-\frac{\mathrm{d}^{2}}{\mathrm{~d} z^{2}}+|\nu| \cosh 2 \mu z
$$


and $f_{\gamma, k}(z)$ be the corresponding solutions of (17).

Thus, to each element $\gamma$ of the dual lattice $\Gamma^{*}$ we associate the functions $\Psi_{\gamma, k}(u, v, z)=$ $e^{2 \pi i(\gamma, w)} f_{\gamma, k}(z)$. The problem with these functions is that they are well defined on the covering space $\tilde{M}^{3}=T^{2} \times \mathbb{R}$ but not on the Sol-manifold $M_{A}^{3}$ itself because they are not invariant with respect to the transformation (1), (2). One can try to construct the genuine eigenfunctions of $\Delta$ on $M_{A}^{3}$ by averaging these functions with respect to the action of $\mathbb{Z}$ on $\tilde{M}^{3}$ generated by this transformation. It turns out that the averaging procedure works.

To show this let us consider instead of $\Psi_{\gamma, k}(u, v, z)$ the following sum

$$
\Phi_{\gamma, k}=\sum_{n \in \mathbb{Z}} \Psi_{\gamma, k}\left(\lambda^{n} u, \lambda^{-n} v, z+n\right)=\sum_{n \in \mathbb{Z}} \Psi_{A^{* n} \gamma, k}(u, v, z) .
$$

Because of the fast decay of the eigenfunctions $f_{\gamma, k}(z)$ this sum is absolutely convergent. It is easy to see that it defines a well-defined function on $M_{A}^{3}$, which is an eigenfunction of the Laplace-Beltrami operator $\Delta$.

The eigenfunctions $\Phi_{\gamma, k}(u, v, z)$ on $M_{A}^{3}$ actually depend only on the orbits $[\gamma]=$ $\left\{A^{* n}(\gamma)\right\}_{n \in \mathbb{Z}}$ with respect to the action of $A^{*}$ on $\Gamma^{*}: \Phi_{\gamma, k}(u, v, z)=\Phi_{[\gamma], k}(u, v, z)$.

We should also consider separately the eigenfunctions related to $\gamma=0$. It is easy to see that the corresponding eigenfunctions have the very simple form

$$
\Phi_{0, s}=1, \cos 2 \pi z, \sin 2 \pi z, \cos 4 \pi z, \sin 4 \pi z, \ldots, \cos 2 k \pi z, \sin 2 k \pi z, \ldots
$$

with the eigenvalues $\mathcal{E}_{k}=(2 \pi)^{2} k^{2}$.

Theorem 2. The eigenfunctions of the Laplace-Beltrami operator $\Phi_{[\gamma], k}(u, v, z),[\gamma] \in$ $\Gamma^{*} \backslash\{0\} / A^{*}$ and $\Phi_{0, s}(z)$ form a complete basis in $L_{2}\left(M_{A}^{3}\right)$.

Proof. The independence and orthogonality of these functions are obvious. The only thing we have to verify is the completeness. To prove this we need to show that any smooth function $\Phi: M_{A}^{3} \rightarrow \mathbb{R}$ which is orthogonal to each eigenfunction from the list is, in fact, zero.

Consider such a function $\Phi(w, z)$ on the covering space $\tilde{M}^{3}$ and expand it as a Fourier series (with respect to $w$ ):

$$
\Phi(w, z)=\sum_{\gamma \in \Gamma^{*}} e^{2 \pi i(\gamma, w)} a_{\gamma}(z)
$$

with some smooth coefficients $a_{\gamma}(z), z \in \mathbb{R}$.

Lemma 1. For all $\gamma \neq 0$ the functions $a_{\gamma}(z)$ have fast decay at infinity and thus belong to $L^{2}(\mathbb{R})$.

Proof. Since $\Phi$ is invariant with respect to the transformation (1), we have $\Phi(w, z)=\Phi(A w, z+1)$. Hence

$$
\sum_{\gamma \in \Gamma^{*}} e^{2 \pi i(\gamma, w)} a_{\gamma}(z)=\sum_{\gamma \in \Gamma^{*}} e^{2 \pi i(\gamma, A w)} a_{\gamma}(z+1)=\sum_{\gamma \in \Gamma^{*}} e^{2 \pi i\left(A^{*} \gamma, w\right)} a_{\gamma}(z+1) .
$$

Thus the Fourier coefficients satisfy the following property:

$$
a_{\gamma}(z+1)=a_{A^{*} \gamma}(z),
$$

or, more generally,

$$
a_{\gamma}(z+n)=a_{A^{* n} \gamma}(z), \quad n \in \mathbb{Z} .
$$


Since the Fourier coefficients $a_{\gamma}$ of a smooth function decay fast for large $\gamma$ and $A^{* k} \gamma$ for $\gamma \neq 0$ tends to infinity we see that the functions $a_{\gamma}(z)$ decay very fast and thus belong to $L^{2}(\mathbb{R})$.

Now suppose that $\Phi(w, z)$ is orthogonal to the eigenfunction $\Phi_{\left[\gamma_{0}\right], k}(u, v, z)=$ $\sum_{n \in \mathbb{Z}} \Phi_{A^{*} \gamma_{0}, k}(u, v, z)$. Since the measure on $M_{A}^{3}$ is proportional to the standard Lebesgue measure $\mathrm{d} u \mathrm{~d} v \mathrm{~d} z$ we have

$$
\begin{aligned}
0 & =\left\langle\Phi(w, z), \Phi_{\left[\gamma_{0}\right], k}(w, z)\right\rangle=\int_{M_{A}^{3}} \Phi(w, z) \bar{\Phi}_{\left[\gamma_{0}\right], k}(w, z) \mathrm{d} \sigma \\
& =\int_{0}^{1}\left(\sum_{\gamma \in \Gamma^{*}} \sum_{n \in \mathbb{Z}} \int_{T^{2}} e^{2 \pi i(\gamma, w)} e^{-2 \pi i\left(A^{* n} \gamma_{0}, w\right)} \mathrm{d} u \mathrm{~d} v\right) a_{\gamma}(z) f_{A^{* n} \gamma_{0}, k}(z) \mathrm{d} z \\
& =\int_{0}^{1}\left(\sum_{n \in \mathbb{Z}} \int_{T^{2}} e^{2 \pi i\left(A^{* n} \gamma_{0}, w\right)} e^{-2 \pi i\left(A^{* n} \gamma_{0}, w\right)} \mathrm{d} u \mathrm{~d} v\right) a_{A^{* n} \gamma_{0}}(z) f_{A^{* n}} \gamma_{0}, k \\
& (z) \mathrm{d} z \\
& =\mathcal{A}\left(T^{2}\right) \int_{0}^{1} \sum_{n \in \mathbb{Z}} a_{A^{* n} \gamma_{0}}(z) f_{A^{* n} \gamma_{0}, k}(z) \mathrm{d} z .
\end{aligned}
$$

We now use the property that $f_{\gamma, k}(z+n)=f_{A^{* n} \gamma, k}(z)$ and $a_{\gamma}(z+n)=a_{A^{* n} \gamma}(z), \quad n \in$ $\mathbb{Z}$ to conclude that

$$
\begin{aligned}
\int_{0}^{1} \sum_{n \in \mathbb{Z}} a_{A^{* n} \gamma_{0}}(z) f_{A^{* n} \gamma_{0}, k}(z) \mathrm{d} z & = \\
\int_{0}^{1} \sum_{n \in \mathbb{Z}} a_{\gamma_{0}}(z+n) f_{\gamma_{0}, k}(z+n) \mathrm{d} z & =\int_{-\infty}^{+\infty} a_{\gamma_{0}}(z) f_{\gamma_{0}, k}(z) \mathrm{d} z .
\end{aligned}
$$

Thus, the Fourier coefficients $a_{\gamma_{0}}(z)$ for $\gamma_{0} \neq 0$ belong to $L^{2}(\mathbb{R})$ and at the same time are orthogonal to all the functions $f_{\gamma_{0}, k}(z)$ which form a complete basis in $L^{2}(\mathbb{R})$. Hence for $\gamma_{0} \neq 0$ the coefficients $a_{\gamma_{0}}(z) \equiv 0$.

This means that the function $\Phi$ must be of the form $\Phi(w, z)=a(z)$, where $a(z)$ is periodic with period 1 . Now using orthogonality to the functions (20) we conclude that $a(z)$ must be identically zero.

Corollary 1. The spectrum of the Laplace-Beltrami operator on Sol-manifolds consists of two parts: the trivial part

$$
\mathcal{E}=\mathcal{E}_{k}=4 k^{2} \pi^{2}, \quad k=0,1, \ldots
$$

corresponding to the eigenfunctions (20) and the non-trivial part

$$
\mathcal{E}=\mathcal{E}_{l,[\gamma]}=\Lambda_{l}(\nu([\gamma]))+\nu([\gamma]) \cos \theta, \quad l=1,2 \ldots, \quad[\gamma] \in \Gamma^{*}\{0\} / A^{*}
$$

related to the modified Mathieu equation (17).

The multiplicities of the trivial eigenvalues are 2 except for the ground state $\mathcal{E}=0$ which has multiplicity 1 . The multiplicities of the non-trivial part of the spectrum are much more interesting and the answer depends on the arithmetical properties of the gluing map $A$. We discuss this in the next section.

\section{Multiplicities of THE EIGENVALUES AND NUMBER THEORY}

As one can see from the previous section the eigenvalue of $\Phi_{[\gamma], k}(u, v, z)$ depends on $\gamma$ only via $Q_{A^{*}}(\gamma)$. Thus the calculation of the multiplicity (for generic values 


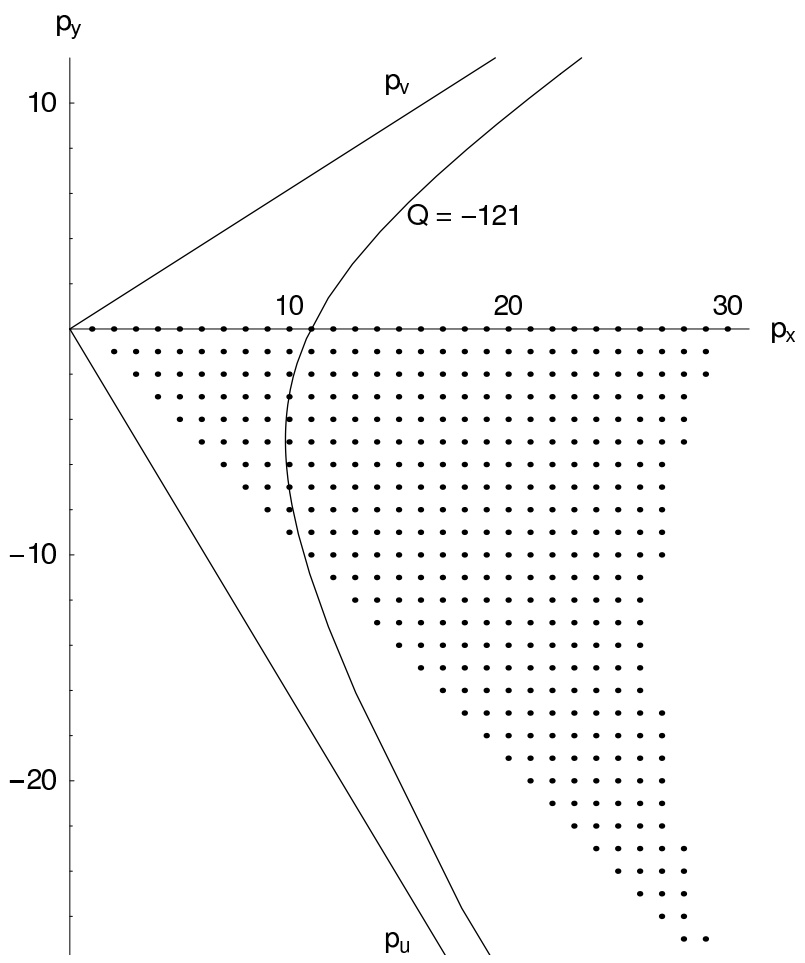

Figure 2. Fundamental domain of the lattice in $\left(p_{x}, p_{y}\right)$ with $|Q| \leq 30^{2}$ for the cat-map. The hyperbola $Q=-11^{2}$ illustrates the first example of a non-trivial degeneracy.

of the parameters to avoid additional accidental coincidences) is reduced to the classical number theoretic problem of finding the number $N_{Q}(n)$ of integer solutions of the equation $Q(x, y)=a x^{2}+b x y+c y^{2}=n$ for a primitive indefinite quadratic form $Q$ different modulo its automorphs. Figure 3 illustrates this for the cat-map $A$ with $Q=-x^{2}+x y+y^{2}$.

For forms $Q$ with certain discriminants there exists an effective formula which allows to compute $N_{Q}(n)$ To be more precise we need the following notion. We say that two forms $Q$ and $Q^{\prime}$ are equivalent if there exists a transformation from $S L(2, \mathbb{Z})$ mapping one into another. It is easy to see that two equivalent forms must have the same discriminant $d=b^{2}-4 a c$. The converse is not true: there can be more than one non-equivalent forms with the same discriminant.

Let $h(d)$ be the number of classes of primitive forms with discriminant $d$. Note that the discriminant $d=b^{2}-4 a c$ is always 0 or 1 modulo 4 and we assume as usual that it is not a total square.

Remark. One should distinguish $h(d)$ and the class number of ideals in the quadratic number field $\mathbf{Q}(\sqrt{d})$. They coincide only if the so-called negative Pell equation

$$
X^{2}-d Y^{2}=-4
$$


has a solution; otherwise $h(d)$ is twice as big (see e.g. [14], Chapter 16). The last property can be reformulated in terms of the period of the continued fraction expansion of $\sqrt{d}$, but a more explicit description is unknown.

If $h(d)=1$ then all forms with the discriminant $d$ are equivalent. In that case there is the following remarkable formula for the number $N_{Q}(n)$ when $n$ is positive and coprime with $d$ :

$$
N_{Q}(n)=N_{d}(n)=\sum_{k \mid n}\left(\frac{d}{k}\right),
$$

where the sum is taken over all divisors of $n$ and $\left(\frac{d}{k}\right)$ is the standard Kronecker symbol (see Landau [18], Chapter IV.4). The Kronecker symbol is a real character modulo $d$, which has the following properties determining it uniquely:

(1) If $d$ and $k$ are not coprime then $\left(\frac{d}{k}\right)=0$;

(2) If $d$ and $k$ are coprime then $\left(\frac{d}{k}\right)= \pm 1$;

(3) $\left(\frac{d}{k l}\right)=\left(\frac{d}{k}\right)\left(\frac{d}{l}\right)$

(4) for $p$ odd prime which is not a divisor of $d\left(\frac{d}{p}\right)$ coincides with the Legendre symbol, which is 1 if $d$ is quadratic residue modulo $p$ and -1 otherwise;

(5) $\left(\frac{d}{2}\right)$ is 1 if $d$ has residue 1 modulo 8 and -1 if it has residue 5 modulo 8 .

For its computation one can use the celebrated Law of Quadratic Reciprocity: if $p, q$ are coprime positive odd numbers then

$$
\left(\frac{p}{q}\right)\left(\frac{q}{p}\right)=(-1)^{\frac{p-1}{2} \frac{q-1}{2}}
$$

Here is the list of the discriminants $d$ up to 100 with $h(d)=1$, see [14]

$$
5,8,13,17,20,29,37,41,52,53,61,65,68,73,85,89,97 .
$$

It is believed that there are infinitely many fundamental discriminants with $h(d)=$ 1 , but it is still an open problem. Notice that for positive definite forms it is known that there are only 9 fundamental discriminants with $h(d)=1$ as it was conjectured by Gauss, namely

$$
d=-3,-4,-7,-8,-11,-19,-43,-67,-163 .
$$

In general if $h(d)>1$ the right-hand side of the formula (21) gives the total number of representations of $n$ by all non-equivalent forms with discriminant $d$. An interesting case is when the ideal class number of $d$ is 1 but $h(d)=2$. In that case we have only two non-equivalent forms with discriminant $d$ : $Q$ and $-Q$ and the formula (21) gives the number of the solutions of the equation $|Q|=n$. The first corresponding discriminants are:

$$
12,21,24,28,32,33,44,45,48,56,57,69,72,76,77,80,84,88,92,93
$$

(see [14]). The only discriminants $<100$ not listed in either table above are $40,60,85,96$ with $h(d)=2,4,2,4$, respectively. Note that most of these discriminants $d$ are not of the form $D=t^{2}-4$, but they can still be obtained from $A \in S L_{2}(\mathbb{Z})$ because $D / d$ may be an arbitrary square.

Now we are ready to describe the multiplicities of the eigenvalues $\mathcal{E}_{l,[\gamma]}$. First we should take into account that the gluing map $A \in S L(2, \mathbb{Z})$ and the corresponding form $Q=Q_{A^{*}}$ may be non-primitive. Let us define the positive integers $r=r(A)$ 
and $l=l(A)$ from the relations $A=A_{0}^{r}$ and $Q_{A^{*}}=l \hat{Q}$, where $A_{0} \in S L(2, \mathbb{Z})$ and $\hat{Q}=\hat{Q}_{A^{*}}$ are primitive.

Theorem 3. The multiplicity $m$ of the eigenvalue $\mathcal{E}_{l,[\gamma]}$ of the Laplace-Beltrami operator $\Delta$ for generic values of the parameters in the metric (3) is

$$
m(\gamma)=2 r(A) N_{Q^{*}}(n),
$$

where $n=Q^{*}(\gamma)=l(A)^{-1} Q_{A^{*}}(\gamma)$. When the discriminant $d$ of the form $Q^{*}$ has class number 1 and $n$ coprime $d$ then $N_{Q^{*}}(n)$ can be computed using the formula (21).

\section{Examples.}

1. Let

$$
A=\left(\begin{array}{ll}
2 & 1 \\
1 & 1
\end{array}\right)
$$

be the so-called cat-map. Then $A^{*}=A$ and $Q_{A}=Q_{A^{*}}=-\left(x^{2}-x y-y^{2}\right)$ are both primitive. The discriminant $D=d=5$ has class number 1 , so one can use the formula $(21)$ to compute the multiplicity of the corresponding $\mathcal{E}_{l,[\gamma]}$. One can check that this leads to the formula

$$
m=2\left(N_{ \pm 1}(n)-N_{ \pm 2}(n)\right),
$$

where $N_{ \pm 1}(n)$ and $N_{ \pm 2}(n)$ are the numbers of divisors of $n=Q_{A}(\gamma)$ which have respectively the residues \pm 1 and \pm 2 modulo 5 .

This example shows that the multiplicities of the eigenvalues can be as big as we like: for example for $n=11^{M}$ the multiplicity is $M+1$; for $n$ a product of $M$ distinct primes all $\pm 1 \bmod 5$ the multiplicity is $2^{M}$.

2. The matrix

$$
A=\left(\begin{array}{cc}
1 & 3 \\
3 & 10
\end{array}\right)
$$

corresponds to $d=13$ with $D=3^{2} d$ and $Q_{A^{*}}=-3\left(x^{2}-3 x y-y^{2}\right)$ so $l(A)=3$.

3. For

$$
A=\left(\begin{array}{ll}
5 & 2 \\
2 & 1
\end{array}\right)
$$

we have $d=8$ with $D=2^{2} d$ and $Q_{A^{*}}=Q_{A}=-2\left(x^{2}-2 x y-y^{2}\right)$ so $l(A)=2$. This example shows that $l(A)$ in general is not directly related to the largest square divisor of $D$.

4. For

$$
A=\left(\begin{array}{ll}
1 & 3 \\
1 & 4
\end{array}\right)
$$

$d=D=21$ and $Q_{A^{*}}=-\left(3 x^{2}+3 x y-y^{2}\right), l(A)=1$. Here $h(d)=2$, but the non-equivalent forms simply differ by a sign.

5. The matrices

$$
A_{1}=\left(\begin{array}{cc}
1 & 6 \\
6 & 37
\end{array}\right) \text { and } A_{2}=\left(\begin{array}{cc}
7 & 18 \\
12 & 31
\end{array}\right)
$$

correspond to $d=40$ with $D=6^{2} d, l\left(A_{i}\right)=6$. Then $Q_{A_{1}^{*}}=-6\left(x^{2}+6 x y-y^{2}\right)$ and $Q_{A_{2}^{*}}=-6\left(3 x^{2}+4 x y-2 y^{2}\right)$ are the two corresponding (non-trivially) non-equivalent forms. 
Remark. In the case when $h(d)$ is larger than 1 in general we do not have a simple formula for the multiplicities for a particular Sol-manifold but only for the disjoint union of Sol-manifolds with non-equivalent forms of given discriminant $d$.

The fact that the multiplicities are large and not sensitive to the change of the parameters in the metric seems to be remarkable. A possible explanation of the rigidity of multiplicities for Sol-manifolds is in the hyperbolicity hidden in the topology of the manifolds.

Remark. The same numbers $N_{Q}(n)$ appear in the harmonic analysis on Solmanifolds as the multiplicities of the irreducible Sol-representations in $C^{\infty}\left(M_{A}^{3}\right)$ (see Chapter 1 in [5]). Although this fact has a similar origin it does not explain the degeneracy of the spectrum of $\Delta$. In fact one can check that that the same degeneracy holds for a more general class of the metrics on $M_{A}^{3}$ :

$$
\mathrm{d} s^{2}=\alpha(z) \mathrm{d} x^{2}+2 \beta(z) \mathrm{d} x \mathrm{~d} y+\gamma(z) \mathrm{d} y^{2}+\mathrm{d} z^{2}
$$

where

$$
\left(\begin{array}{cc}
\alpha(z) & \beta(z) \\
\beta(z) & \gamma(z)
\end{array}\right)=\exp (-z B)^{\top}\left(\begin{array}{cc}
\alpha_{0}(z) & \beta_{0}(z) \\
\beta_{0}(z) & \gamma_{0}(z)
\end{array}\right) \exp (-z B) .
$$

Here $\exp B=A$ and $\alpha_{0}(z), \beta_{0}(z), \gamma_{0}(z)$ are arbitrary real 1-periodic functions with the only condition that the form $\mathrm{d} s^{2}=\alpha_{0}(z) \mathrm{d} x^{2}+2 \beta_{0}(z) \mathrm{d} x \mathrm{~d} y+\gamma_{0}(z) \mathrm{d} y^{2}$ is positive definite for all $z$. The Sol-invariant metrics (3) correspond to the case when these functions are constant. The degeneracy in this case follows again from the separation of variables and thus is not directly related to the $S o l$-invariance.

It is interesting to compare the $S o l$-case with the spectra of flat tori $T^{2}$. It is easy to see that in the last case the answer will depend drastically on the metric parameters (or equivalently, on the geometry of the basic parallelogram). For example, if it is a square then the spectrum up to a multiple is given by the values of the standard quadratic form $n=x^{2}+y^{2}$, and the multiplicity are given by the Gauss' famous formula

$$
m=4\left(N_{1}(n)-N_{3}(n)\right),
$$

where $N_{1}(n)$ and $N_{3}(n)$ are the numbers of the divisors of $n$ with the residues 1 and 3 modulo 4 respectively. If however the basic parallelogram is generic then all multiplicities are 2 (which is due to the central symmetry of the problem).

\section{Semiclassical analysis AND Weyl's LAW}

It is instructive to see how our exact calculation of the spectrum agrees with the famous Weyl's law [29], which says that for a quantum system the number $N(\Lambda)$ of the eigenvalues $\mathcal{E} \leq \Lambda$ for large $\Lambda$ asymptotically is equal (up to a factor $(2 \pi)^{-n}$ ) to the volume of the domain in the classical phase space with the energy less than $\Lambda$. For our Laplace-Beltrami operator (15) this means that

$$
N(\Lambda) \sim \frac{4}{3} \pi \Lambda^{3 / 2} \frac{\operatorname{Vol}\left(M_{A}^{3}\right)}{(2 \pi)^{3}}=\frac{4}{3} \pi \Lambda^{3 / 2} \frac{\mathcal{A}\left(T^{2}\right)}{(2 \pi)^{3}},
$$

where $\operatorname{Vol}\left(M_{A}^{3}\right)$ is the volume of our Sol-manifold (which equals the area of the fibre $\mathcal{A}\left(T^{2}\right)$ since the length in $z$-direction was assumed to be 1$)$.

Let us count now the eigenvalues $\mathcal{E}$ using the results of section 5 . Let us assume for simplicity that $\cos \theta=0$, so besides the trivial part they coincide with the 
eigenvalues of the Mathieu operator

$$
\mathcal{M}=-\frac{\mathrm{d}^{2}}{\mathrm{~d} z^{2}}+|\nu| \cosh 2 \mu z
$$

where as before

$$
\nu=\frac{8 \pi^{2} Q}{\sqrt{D} \mathcal{A}\left(T^{2}\right) \sin \theta}
$$

and $Q$ is the corresponding binary quadratic form.

First of all let us use the well-known fact from number theory (see e.g. [14]) that for large $Q_{0}$ the number of lattice points (modulo $A$ ) with values of $|Q|$ less than $Q_{0}$ is proportional to the area of the fundamental domain up to $Q_{0}$ :

$$
M\left(Q_{0}\right) \sim 4 \frac{\mu}{\sqrt{D}} Q_{0}
$$

The factor of four counts lattice points related by the symmetry given by changing the sign of both $p_{u}$ and $p_{v}$ and also accounts for the states in the quadrants where $Q$ is of opposite sign.

For fixed value of $Q$ (hence $\nu$ ) there is a whole line of eigenvalues of the Mathieu operator (24). The number of these eigenvalues up to energy $\Lambda$ for large $\Lambda$ is given asymptotically by the action integral

$$
I(\Lambda, Q)=\frac{1}{2 \pi} \oint \sqrt{\Lambda-|\nu| \cosh 2 \mu z)} d z,
$$

which is of course the area of the domain in the phase plane with energy less than $\Lambda$ divided by $2 \pi$. This can be simplified to

$$
2 \pi \mu I=\sqrt{\Lambda} \oint \sqrt{1-g \cosh 2 \zeta} \mathrm{d} \zeta, \quad g=|\nu| / \Lambda
$$

With $\xi=\cosh (2 \zeta)$ this becomes a standard elliptic integral (see e.g. [30])

$$
\frac{2 \pi \mu}{\sqrt{\Lambda}} I=2 \int_{1}^{1 / g} \frac{\sqrt{1-g \xi}}{\sqrt{\xi^{2}-1}} \mathrm{~d} \xi=4 \sqrt{1+g}(K(k)-E(k)), \quad k^{2}=\frac{1-g}{1+g} .
$$

Let us denote this expression $f(g)$.

Thus we see that the total number of states up to energy $\Lambda$ is

$$
N(\Lambda) \sim \int M^{\prime}(Q) I(\Lambda, Q) \mathrm{d} Q=2 \frac{\Lambda^{3 / 2}}{C \mu \pi} \int_{0}^{1} \frac{\mu}{\sqrt{D}} f(g) \mathrm{d} g,
$$

where $C=\frac{8 \pi^{2}}{\sqrt{D} \mathcal{A}\left(T^{2}\right) \sin \theta}$. The integral over $g$ is best performed by treating it as a double integral over $g$ and $\xi$. Introducing $\eta=g \xi$ and performing the $\eta$-integral first gives

Thus we have

$$
\int_{0}^{1} f(g) \mathrm{d} g=\frac{4}{3} \int_{1}^{\infty} \frac{\mathrm{d} \xi}{\xi \sqrt{\xi^{2}-1}}=\frac{2}{3} \pi
$$

$$
N(\Lambda) \sim \Lambda^{3 / 2} \frac{\mathcal{A}\left(T^{2}\right)}{(2 \pi)^{3}} \frac{4 \pi}{3} \sin \theta
$$

which agrees with Weyl's formula (23) when $\theta=\pi / 2$.

For general $\theta$ we have $\mathcal{E}=\lambda-\nu \cos \theta$. In that case we need to compute

$$
X_{ \pm}=\iint \sqrt{1-g(\cosh 2 z \pm \cos \theta)} \mathrm{d} z \mathrm{~d} g
$$




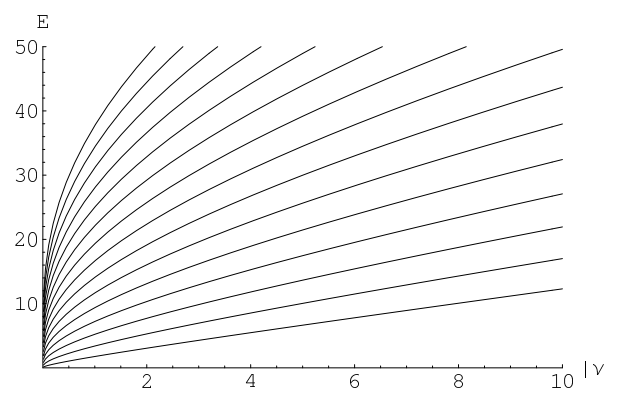

FigurE 3. First 15 states of the cosh-Mathieu equation in dependence of the parameter.

over the domain $0 \leq g \leq 1 /(1 \pm \cos \theta)$ and $|z| \leq z_{0}$ where $z_{0}$ is the smallest positive root of the integrant. The transformation

$$
\eta=g(\cosh 2 z+\cos \theta), \quad \xi=\cosh 2 z
$$

folds the integration region to the rectangle $\xi>1$ and $0 \leq \eta \leq 1$ and the integral becomes

$$
X_{ \pm}=2 \iint \frac{\sqrt{1-\eta}}{(\xi \pm \cos \theta) \sqrt{\xi^{2}-1}} \mathrm{~d} \eta \mathrm{d} \xi .
$$

The integral over $\eta$ is easily done as before while the integral over $\xi$ evaluates to

$$
X_{ \pm}=\frac{4}{3} \frac{\frac{\pi}{2} \pm\left(\theta-\frac{\pi}{2}\right)}{\sin \theta} .
$$

Hence the sum of the contributions from the two cases of positive and negative $Q$ is

$$
\sin \theta\left(X_{+}+X_{-}\right)=\frac{4}{3} \pi
$$

as before. This computation shows that $\theta$ determines the relative number of states between the regions with positive and negative $Q$, namely $X_{+} / X_{-}=\theta /(\pi-\theta)$.

Let us look what this calculation gives for the first eigenvalues of $\Delta$. There are two opposite cases depending on whether geometric parameter $A=\mathcal{A}\left(T^{2}\right) \sin \theta$ is small or large. Let us assume again for simplicity that $\theta=\pi / 2$, then $A$ is simply the area of the fibre.

The small $A$ corresponds to the "rope-like" Sol-manifolds. In this case the first eigenvalues are "trivial": $\mathcal{E}_{k}=4 k^{2} \pi^{2}, \quad k=0,1,2 \ldots$ which correspond to $Q=0$.

The second case when $A$ is large is more interesting. In that case the parameter $\nu$ in the Mathieu operator is small. The action integral (26) for small $\nu$ has the $\operatorname{asymptotics}^{1}$

$$
I \sim \frac{\sqrt{\Lambda}}{\mu \pi} \ln \frac{\Lambda}{|\nu|} .
$$

This suggests the following asymptotics for the eigenvalues for small $\nu$ :

$$
\Lambda_{k}=\frac{(\mu \pi k)^{2}}{(\ln |\nu|)^{2}}, k=0,1,2 \ldots
$$

\footnotetext{
${ }^{1}$ The fact that the action diverges logarithmically for $Q \rightarrow 0$ seems to be surprising. To our knowledge this is the first example of Liouville integrable system on a compact manifold for which the action diverges on approach of a singular level (with energy fixed).
} 
Note that although $\Lambda_{k} \rightarrow 0$ as $\nu \rightarrow 0$ the decay is slower than any power of $\nu$. The first 15 states are shown in fig. 3.

For the corresponding Sol-manifolds this gives the following behaviour of the first eigenvalues

$$
\mathcal{E}_{k,[\gamma]} \sim \frac{(\mu \pi k)^{2}}{\left(\ln \left|C Q_{A^{*}}([\gamma])\right|\right)^{2}}, \quad k=0,1,2, \ldots, \quad[\gamma] \in \Gamma^{*} \backslash\{0\} / A^{*}
$$

for small $C=\frac{8 \pi^{2}}{\sqrt{D} \mathcal{A}\left(T^{2}\right) \sin \theta}$. If we order these eigenvalues $\mathcal{E}_{0}=0 \leq \mathcal{E}_{1} \leq \mathcal{E}_{2}, \ldots$ then we have

$$
\mathcal{E}_{j} \sim \frac{(\mu \pi)^{2}}{(\ln C)^{2}}\left(1-2 \frac{\ln Q_{j}}{\ln C}\right), \quad j=1,2, \ldots,
$$

where $Q_{0}=0 \leq Q_{1} \leq Q_{2}, \ldots$ are positive values of the form $Q_{A^{*}}$ listed in increasing order and we have assumed that $Q_{j} \ll \frac{1}{C}$. In particular,

$$
\mathcal{E}_{1} \sim \frac{(\mu \pi)^{2}}{(\ln C)^{2}}, \quad \frac{\mathcal{E}_{j}-\mathcal{E}_{1}}{\mathcal{E}_{i}-\mathcal{E}_{1}} \sim \frac{\ln \left(Q_{j} / Q_{1}\right)}{\ln \left(Q_{i} / Q_{1}\right)},
$$

so when $C$ is small we "see" the values of the quadratic form $Q_{A^{*}}$ straight from the spectrum.

Note that the question about the next order term in Weyl's law is non-trivial. For the simpler case of $N i l$-manifolds some results in this direction can be found in $[20]$.

One can also look at the corresponding Minakshisundaram-Plejel asymptotic expansion, which is an important characteristics of the spectra (see e.g.[10]). In particular the second coefficient in this expansion is proportional to the integral of the scalar curvature $K$. A straightforward calculation shows that for the Sol-manifold $M_{A}^{3}$ the principal sectional curvatures are $\pm \sin 2 \theta \log ^{2} \lambda$ and $-2(\sin \theta \log \lambda)^{2}$, so

$$
K=-2(\sin \theta \log \lambda)^{2}
$$

and thus is always negative.

\section{Spectral Statistics}

The spectral statistics of integrable and chaotic systems is quite different, see, e.g., $[2,3,9]$. As we have seen the geodesic flow on Sol-manifolds has properties of both, integrable and chaotic systems. Therefore it is a natural question what the spectral statistics of the Sol-manifolds is like. Note that according to the Berry-Tabor conjecture [2] integrable systems should have Poisson distributed level spacing. We are going to show that this is not the case for Sol-manifolds.

The reason is the high multiplicities of the eigenvalues. Indeed, for the simplest positive quadratic form $Q_{0}=x^{2}+y^{2}$ the classical result due to E. Landau says that the number of integers up to a number $K$ represented by this form grows as $K / \sqrt{\log K}$. If there would be no degeneracies then this number would grow like the area of the fundamental region, which is proportional to $K$. This means that most of the level spacings of the values of $Q_{0}$ are zero.

According to P. Sarnak [22, 23] a similar fact is true for indefinite forms as well, namely

The number of positive integers up to $K$ that can be represented by a given indefinite quadratic form $Q$ grows not faster than $O(K / \sqrt{\log K})$. 

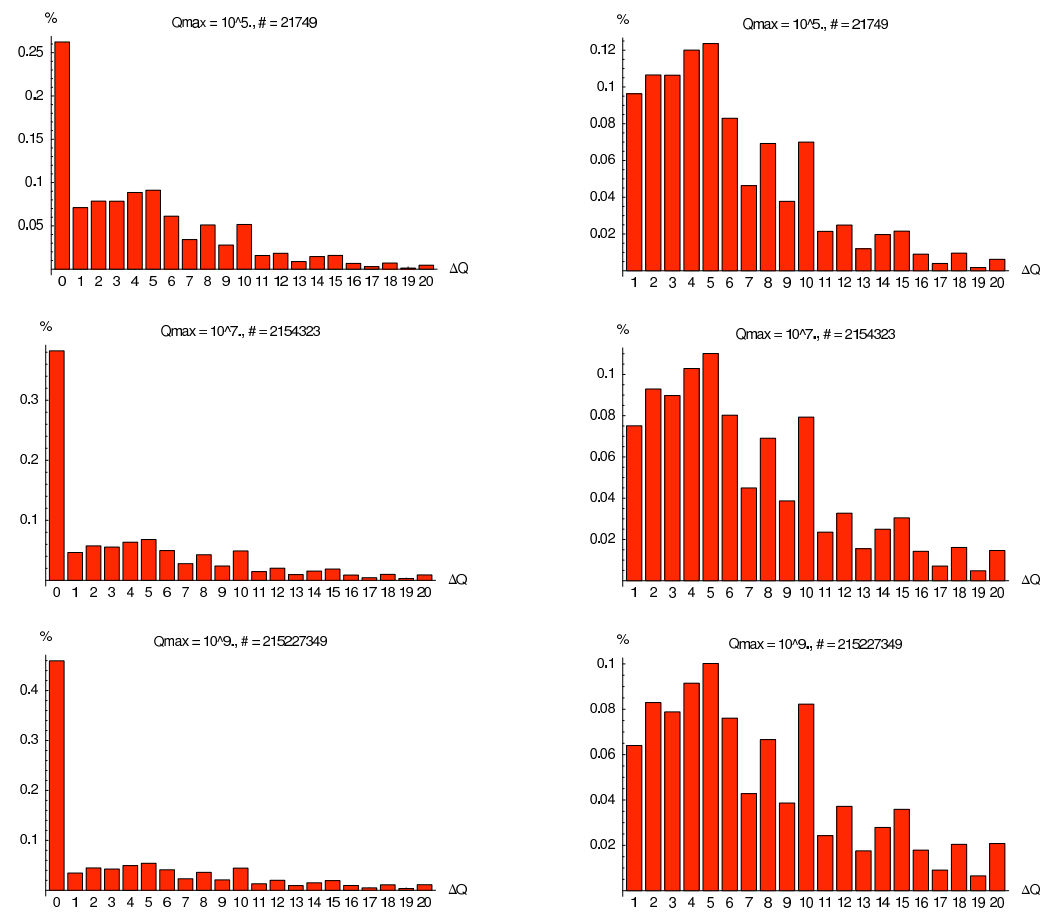

FIGURE 4. Level spacing statistics of the indefinite binary quadratic form $Q(x, y)=-x^{2}+x y+y^{2}$ (left) with degeneracies removed (right)

Combining this with the results of section 5 we have the following

Theorem 4. The level spacing distribution for the spectrum of Sol-manifolds $M_{A}^{3}$ is not Poisson and hence the Berry-Tabor conjecture does not hold in this case.

This is particularly interesting because this statement is not sensitive to change of the metric in the Sol-class (3) (or even more general class (22)).

Let us illustrate this in the example of the cat-map $A$. In fig. 4 (left) the level spacing statistics for the indefinite binary quadratic form $Q_{A}=-x^{2}+x y+y^{2}$ is shown for three different values of $Q_{\max }$.

Since the cat-map is the product of two involutions, there is a simple reflection symmetry in the lattice, which causes almost all states to be at least twofold degenerate. This discrete symmetry needs to be factored out before the level spacing statistics can be studied. The involutions $R_{i}$ with $R_{i}^{2}=I d$ are

$$
A=R_{2} R_{1}, \quad R_{1}=\left(\begin{array}{cc}
1 & 0 \\
-1 & -1
\end{array}\right), \quad R_{2}=\left(\begin{array}{ll}
1 & -1 \\
0 & -1
\end{array}\right) .
$$

The fixed line of $R_{1}$ is the line $y=-x / 2$, and factoring the fundamental region in fig. 2 by $R_{1}$ simply cuts the fundamental region in half along this line. Since the values of $Q$ are integers we chose to present the raw level spacing statistics, i.e. without unfolding the spectrum first. The number of lattice points found up to the corresponding $Q_{\max }$ in the reduced fundamental region is given in the heading of each figure, and the ratio approaches $\ln \lambda /(2 \sqrt{d})$. The figures clearly show that 

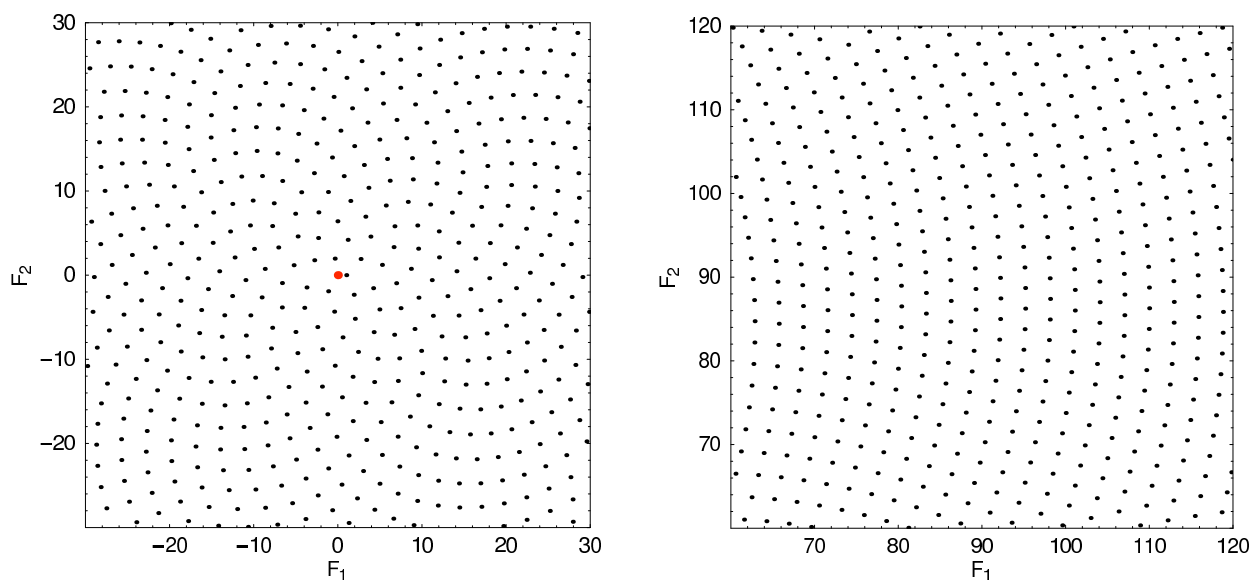

FiguRE 5. Image of the lattice $\mathbb{Z}^{2}$ in $\left(p_{x}, p_{y}\right)$ under the momentum map $\left(F_{1}, F_{2}\right)$ for fixed energy where $A$ is the cat-map. Left: origin at the centre. Right: distorted standard lattice away from the origin.

the proportion of degenerate levels grows in agreement with what we said above. When the degenerate levels are discarded in the statistics fig. 4 (right) shows that the distribution appears to converge to some non-universal shape. We would like to mention here the paper [13] where the moments of the intervals between the sums of two squares were studied.

\section{QUANTUM MONODROMY}

In view of the previous results the appearance of quantum monodromy in our problem is quite natural. However there is a problem with this notion in our case which we want to discuss first.

As it was shown in [4] the geodesic flow on Sol-manifolds can not have three analytic integrals (see Section 3 above). A similar fact holds in the quantum case. Namely, one can show that the algebra of the differential operators on the Solmanifold $M_{A}^{3}$ commuting with the Laplace-Beltrami operator $\Delta(15)$ is generated by $\Delta$ and $\frac{\partial}{\partial u} \frac{\partial}{\partial v}$. This means that our quantum problem does not have enough quantum integrals, at least in the class of the differential operators and therefore it is not clear if we can apply the rigorous treatment of quantum monodromy from [27].

So in this section we will treat the quantum monodromy on Sol-manifolds on the intuitive level paying more attention to geometry rather than to analysis. As we have already shown, the set of eigenfunctions is in a natural one-to-one correspondence with $\Gamma^{*} / A^{*} \times \mathbb{N},[\gamma] \in \Gamma^{*} / A^{*}, k \in \mathbb{N}$. The fundamental domain of $A^{*}$ is shown in fig. 2. It is natural to represent the orbit space as a lattice on the cone obtained by gluing the edges of the fundamental domain of $A^{*}$ on the plane (more precisely we should consider four different cones corresponding exactly to four families of Liouville tori).

Quantum monodromy arises when we pass around the vertex of the cone. It is clear that the basis of the lattice will undergo the transformation $A$. On the 

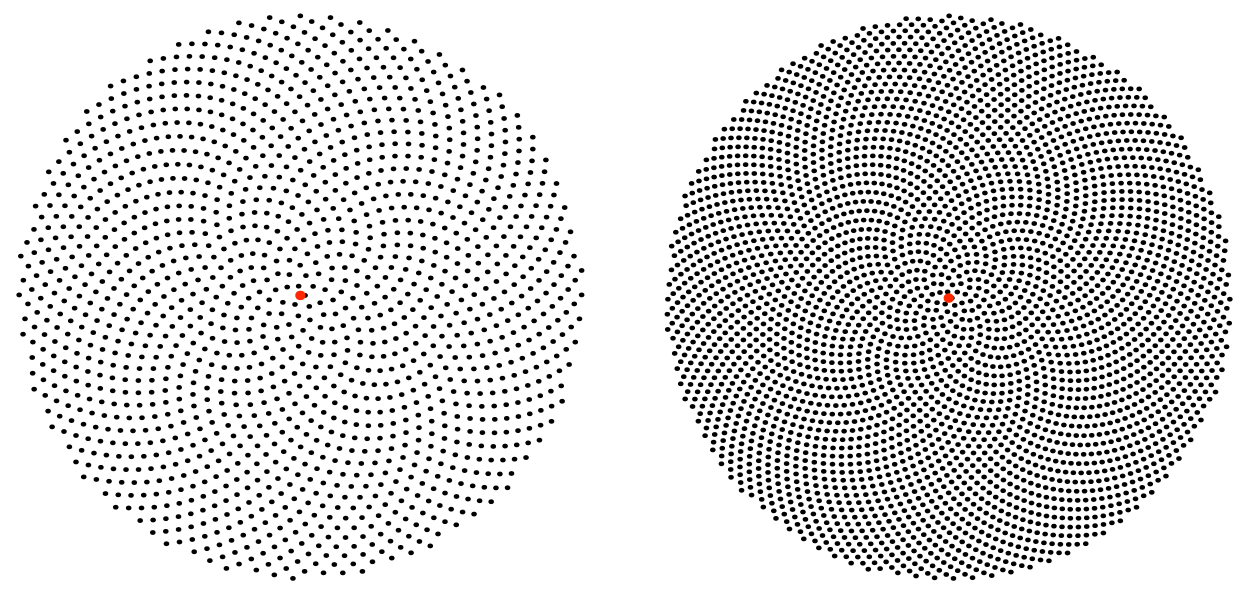

Figure 6. Image of the lattice $\mathbb{Z}^{2}$ in $\left(p_{x}, p_{y}\right)$ under the momentum map $\left(F_{1}, F_{2}\right)$ for fixed energy and $A$ the cat-map. Left: Sol-flower with $|Q| \leq 60^{2}$. Right: Sol-flower with $|Q| \leq 90^{2}$

other hand, nothing happens to the third direction corresponding to the parameter $k$. Therefore the quantum monodromy for the Sol-manifold $M_{A}^{3}$ is given by the matrix

$$
\left(\begin{array}{cc}
A^{*} & 0 \\
0 & 1
\end{array}\right)
$$

We want to emphasize that in this case quantum monodromy has a purely topological nature. It is determined by the topology of the underlying manifold, and not by properties of the metric. It does not depend on the parameters $E, G, F$, moreover the monodromy remains the same for all metrics of the form

$$
\mathrm{d} s^{2}=\mathrm{d} s_{z}^{2}+\mathrm{d} z^{2}
$$

where $\mathrm{d} s_{z}^{2}$ is a flat metric on fibres $T_{z}^{2}$ with coefficients depending on $z$. In the previously known examples (like the geodesic flow on the 3-dimensional ellipsoid of revolution, [28]) the metric $g$ played the principal role.

In figures 5,6 , and 7 we demonstrate the quantum monodromy of the Solmanifold $M_{A}^{3}$ related to the cat-map

$$
A=\left(\begin{array}{ll}
2 & 1 \\
1 & 1
\end{array}\right)
$$

To make the image of the lattice uniform we have slightly modified the classical integrals $f_{1}, f_{2}$ from section 3 as follows:

$$
\begin{aligned}
& F_{1}=\sqrt{|Q|} \cos 2 \pi \beta, \\
& F_{2}=\sqrt{|Q|} \sin 2 \pi \beta,
\end{aligned}
$$

where $Q=p_{u} p_{v}$ and $\beta=\frac{\ln \left|p_{u}\right|}{\ln \lambda}$. The image of the lattice under the map $F$ we call Sol-flower (see fig. 6). Note that an alternative choice $\beta=\frac{\ln \left|p_{v}\right|}{\ln \lambda}$ would give a similar picture and the freedom of the rescaling of the eigenvectors $e_{u}, e_{v}$ leads simply to a rotation of the plane $\left(F_{1}, F_{2}\right)$. Fig. 5 illustrates that away from the origin the lattice is simply a deformed standard lattice. 

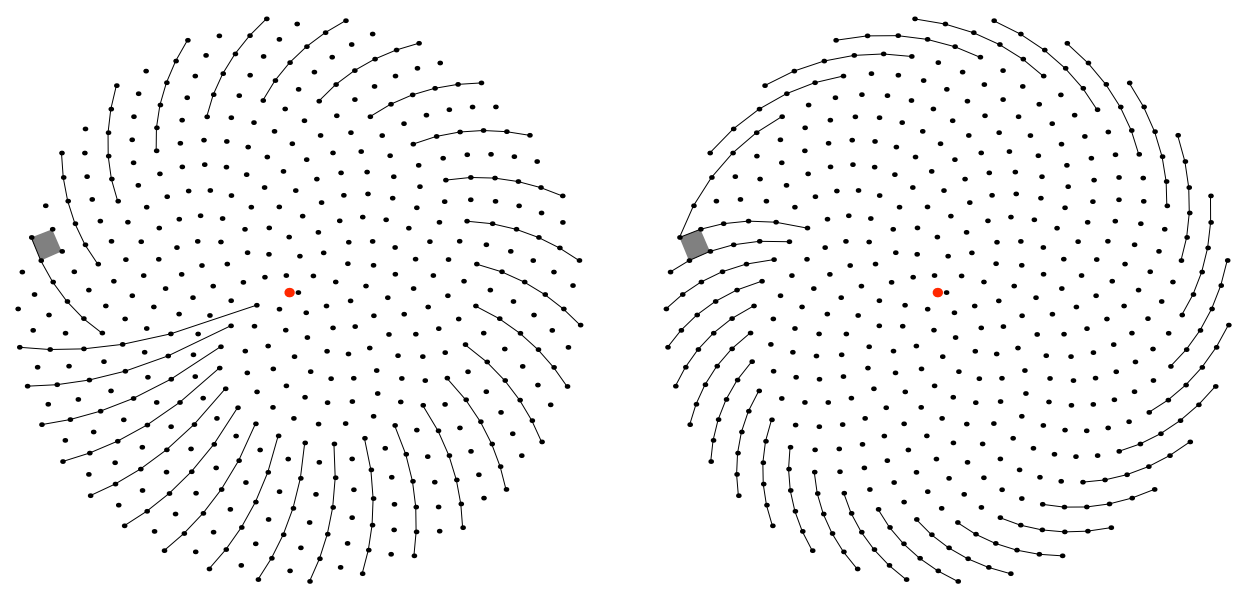

FiguRE 7. Parallel transport of basic directions in the image of the momentum map.

A nice property of the map $\left(p_{u}, p_{v}\right) \rightarrow\left(F_{1}, F_{2}\right)$ is that it changes the area simply by a constant multiple: it is easy to check that

$$
\mathrm{d} F_{1} \wedge \mathrm{d} F_{2}=\frac{\pi}{\ln \lambda} \mathrm{d} p_{u} \wedge \mathrm{d} p_{v} .
$$

It is interesting to mention that the multiplicity problem becomes the standard "circle problem" if one replaces the square lattice by the Sol-flower (but of course it does not help to compute them).

When a fundamental cell is chosen in the Sol-flower as indicated in grey in fig. 7 the monodromy can be observed as follows: A line extending a basis vector is parallel transported in the lattice. After completing a cycle about the origin this direction is changed. The left picture shows images of the lines $\left(p_{x}, p_{y}\right)=$ $(30-2 l,-j-l), j=0 . .27, l=0 . .5$. The right picture shows images of the lines $\left(p_{x}, p_{y}\right)=(30-l,-j-l), j=0 . .30, l=0 . .5$. Denote the direction of the line shown in the left part of fig. 7 by $e_{1}$, and the one on the right part by $e_{2}$. The preimages of these basis vectors in fig. 2 are $-\left(2 e_{x}+e_{y}\right)$ and $-\left(e_{x}+e_{y}\right)$. Parallel transporting $e_{1}$ clockwise by increasing $j$ gives $e_{1}+e_{2}$ (determining the second row of $A$ ), while parallel transporting $e_{2}$ counterclockwise by decreasing $j$ gives $-e_{1}+e_{2}$ (determining the first row of $A^{-1}$ ). Since $A \in S L(2, \mathbb{Z})$ this determines the cat-map.

\section{Concluding Remarks}

The Sol-geometry from a dynamical point of view has the special property of being on the border between integrability and chaos. Integrability is reflected in the solvability of the corresponding group while the chaos is related to a hidden (partial) hyperbolicity. This makes the Sol-case of particular interest and explains why the geodesic problem on the Sol-manifolds has both integrable and chaotic features. As we have seen the quantum case gives a new interesting twist to the story by bringing arithmetic into play.

Atiyah, Donelly and Singer [1] considered a more general case of Sol-manifolds which are $T^{n+1}$ torus fibres over $T^{n}$. Much of our analysis can be generalised to 

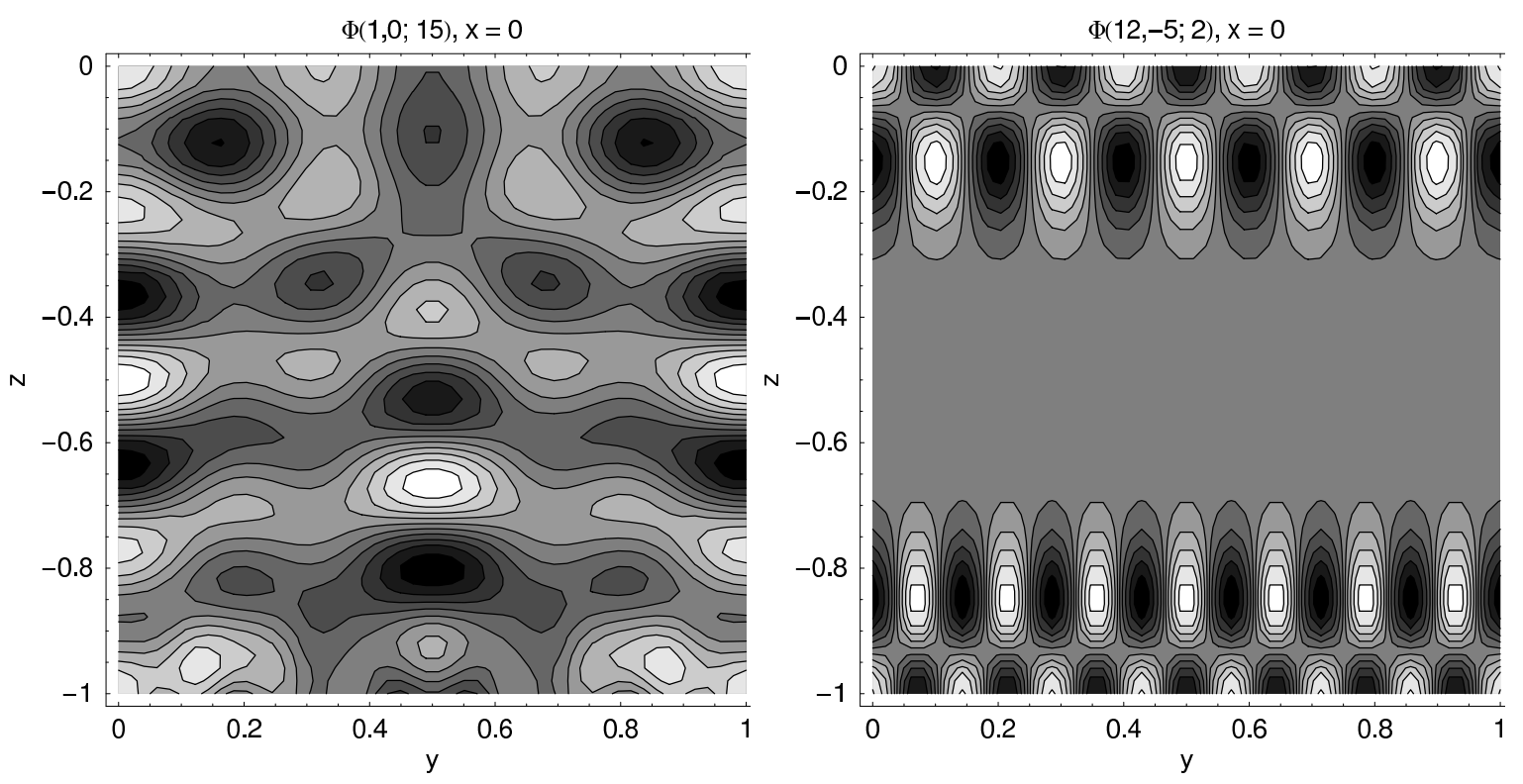

Figure 8 . Slice of $\Re\left(\Phi_{[\gamma], k}\right)$ at $x=0$ for $\gamma=(1,0), k=15$ (left) and $\gamma=(12,-5), k=2$ (right) for the cat-map. The appearance of the eigenfunction whose $Q(\gamma)$ is small and thus close to the classical chaos is more irregular.

this case as well. The quantum Toda lattice Hamiltonian will appear then as a generalisation of the modified Mathieu operator. Some very interesting results in the corresponding classical problem were found recently by Leo Butler in [6].

It would be also interesting to study in more detail how the chaos (at the degenerate level $Q=0$ ) of the classical system manifests itself in the quantum version. We showed that the spectral statistics provides a counterexample to the Berry-Tabor conjecture, but it cannot be taken as an indicator of chaos. One simple observation is that the trivial eigenfunctions $\Phi_{0, s}$ are asymptotically 'uniformly distributed' on the manifold. Hence the subset of eigenfunctions that are associated with the classical chaos are quantum unique ergodic, cf. [17]. Already at relatively small quantum numbers it can be seen that the nodal lines are more complicated when $Q$ is small, see fig. 8 .

\section{ACKNOWLEDGEMENTS}

We are very grateful to M. Berry, E. Bombieri, V. Kuznetsov, J. Marklof, A. Pushnitski, P. Sarnak, R. Schubert and P. Shiu for very useful and stimulating discussions.

This work has been started in December 2002 when one of us (A.B.) visited Loughborough University. We are grateful to the London Mathematical Society for the support of this visit.

\section{REFERENCES}

[1] M.F. Atiyah, H. Donnelly, I.M. Singer Eta invariants, signature defects of cusps and values of L-functions. Ann. Math. 118 (1983), 131-177. 
[2] M.V. Berry and M. Tabor Level clustering in the regular spectrum. Proc. Roy. Soc. A 356 (1977), 375-394.

[3] O. Bohigas, M.-J. Giannoni and C. Schmidt Characterization of chaotic quantum spectra and universality of level fluctuation laws. Phys. Rev. Lett. 52 (1984), 1-4.

[4] A.V. Bolsinov, I.A. Taimanov Integrable geodesic flows with positive topological entropy. Invent. Math. 140 (2000), 639-650.

[5] J. Brezin, Harmonic analysis on compact solvmanifolds, LNM 602, (1977)

[6] L. Butler The Toda lattice, positive-entropy integrable systems and transcendental numbers. Preprint, 2003.

[7] R. Cushman and J.J. Duistermaat The quantum spherical pendulum. Bull. Am.Math. soc. (N.S.) 19 (1988), 475-479.

[8] J.J. Duistermaat, On global action-angle coordinates, Comm. Pure Appl. Math., 33, (1980), 687- 706

[9] J.J.Duistermaat, V.W. Guillemin. The spectrum of positive elliptic operators and periodic bicharacteristics. Invent. Math. 29 (1975), no. 1, 39-79.

[10] P. Gilkey Spectral geometry of a Riemannian manifold. J. Diff. Geom. 10, n.4, 601- 618.

[11] V. Guillemin and A. Uribe Monodromy in the quantum spherical pendulum. Comm. Math. Phys. 122 (1989), 563-574.

[12] F. Hirzebruch Hilbert modular surfaces. L'Enseign.Math. 19 (1973), 183-281.

[13] C. Hooley On the intervals between numbers that are sums of two squares. Acta Math. 127 (1971), 279-297.

[14] Hua Loo Keng Introduction to Number Theory. Springer Verlag, 1982.

[15] I.V. Komarov, L.I. Ponomarev and S.Yu. Slavyanov Spheroidal and Coulomb spheroidal functions. "Nauka", Moscow, 1975. 320 pp (Russian).

[16] I.V. Komarov, A.B. Tsiganov Quantum two-particle periodic Toda lattice. Vestnik LGU, Ser. 4, N. 2 (1988), 69-71 (Russian).

[17] D. Jakobson, N. Nadirashvili, J. Toth Geometric properties of eigenfucntions Russian Maths. Surveys 56 (2001) 1085-1105

[18] E. Landau Elementary Number Theory. Chelsea, New York, 1958.

[19] W.J. LeVeque Fundamentals of Number Theory. Dover Publications, New York, 1996.

[20] Y.N. Petridis, J.A. Toth The remainder in Weyl's law for Heisenberg manifolds. J. Differential Geom. 60 (2002), 455-483.

[21] P. Sarnak Class numbers of indefinite binary quadratic forms. J. Number Theory 15 (1982), 229-247.

[22] P. Sarnak Values at integers of binary quadratic forms. CMS Conf. Proc., 21, Amer. Math. Soc., Providence, RI, (1997), 181-203.

[23] P. Sarnak Private communication (June 2003).

[24] P. Scott The geometries of 3-manifolds. Bull. London Math. Soc., 15 (1983), 401-487.

[25] I.A. Taimanov Topological obstructions to integrability of geodesic flows on non-simplyconnected manifolds. Math. USSR Izv. 30 (1988), 403-409.

[26] W.P. Thurston Hyperbolic geometry and 3-manifolds. London Math. Soc. Lecture Note Ser., 48, Cambridge Univ. Press, 1982.

[27] Vu Ngoc San Quantum monodromy in integrable systems. Comm. Math. Phys. 203 (1999), no. 2, 465-479.

[28] H. Waalkens, H.R. Dullin, Quantum monodromy in prolate ellipsoidal billiards, Ann. Physics, 295 (2002), $81-112$

[29] H. Weyl, Das asymptotische Verteilungsgesetz der Eigenwerte linearer partieller Differentialgleichungen Math. Ann. 141 (1912) 441 - 479.

[30] E.T. Whittaker and G.N. Watson A Course in Modern Analysis, 4th ed., Cambridge University Press, 1990.

[31] D. Zwillinger Handbook of Differential Equations, 3rd ed. Boston, MA: Academic Press, 1997. 
Department of Mathematics and Mechanics, Moscow State University, 119899 Moscow, Russia

E-mail address: bolsinov@mech.math.msu.su

Department of Mathematical Sciences, Loughborough University, Loughborough, LEICESTERSHIRE, LE11 3TU, UK

E-mail address: H.R.Dullin@lboro.ac.uk

Department of Mathematical Sciences, Loughborough University, Loughborough, Leicestershire, LE11 3TU, UK and Landau Institute for Theoretical Physics, Moscow, Russia

E-mail address: A.P.Veselov@lboro.ac.uk 